\title{
Regularity properties of the diffusion coefficient for a mean zero exclusion process
}

\author{
M. Sued \\ IMPA: Estrada Dona Castorina 110, Rio de Janeiro, Brazil \\ Received 5 March 2003; received in revised form 11 November 2003; accepted 11 February 2004 \\ Available online 11 September 2004
}

\begin{abstract}
The asymmetric mean zero simple exclusion process is an example of non-reversible nongradient system. We prove that the diffusion coefficient of its hydrodynamic equation is a $C^{\infty}$ function on $[0,1]$, in all dimension.

() 2004 Elsevier SAS. All rights reserved.

\section{Résumé}

Le processus d'exclusion simple asymétrique de moyenne nulle est un exemple de modèle non-réversible nongradient. Nous démontrons que le coefficient de diffusion de l'équation hydrodynamique est une fonction $C^{\infty}$ sur $[0,1]$, en toute dimension. (c) 2004 Elsevier SAS. All rights reserved.
\end{abstract}

Keywords: Mean zero exclusion process; Regularity of the diffusion coefficient

\section{Introduction}

The simple exclusion process represents the evolution of particles on the lattice $\mathbb{Z}^{d}$ with a hard-core interaction that prevents more than one particle per site. The evolution can be informally described as follows: each particle waits a mean one exponential time. When the clock rings, it chooses a site to jump. The probability that a particle located at $x$ picks the site $y$ is given by $p(y-x)$, were $p$ a probability measure in $\mathbb{Z}^{d}$. If the chosen site is free, the particle jumps. Otherwise it remains in its place and waits for a new exponential time. All the particles are performing this, independently one of each other. In this work we focus our attention in the finite range $(p(x)=0$ for $\|x\|$ big enough) mean zero case: $\sum x p(x)=0$.

E-mail address: msued@impa.br (M. Sued). 
Under diffusive scaling, the hydrodynamic equation for the mean zero simple exclusion process evolving in the torus is described by the non-linear parabolic differential equation

$$
\partial_{t} \rho=\sum_{i, j=1}^{d} \partial_{u_{i}}\left\{D_{i, j}(\rho) \partial_{u_{j}} \rho\right\} .
$$

This result was obtained by Xu in dimension 1 [14] extending to the non-reversible setting the nongradient method developed by Varadhan [12] and Quastel [10].

The main result of this work is that the coefficients $\left\{D_{i, j}(\alpha), 1 \leqslant i, j \leqslant d\right\}$ are smooth functions in $\alpha$. This fact guarantees the existence of regular solutions for the hydrodynamic equation and permits the derivation of weak conservation of local equilibrium through the relative entropy method [15]. We extend, in particular, Xu's result to dimension $d \geqslant 2$. Furthermore, since the system is attractive, good dependence on the initial condition for the solution of the hydrodynamic equation allows to prove conservation of local equilibrium [4].

The method used to prove regularity of the diffusion coefficient was developed by Landim, Olla and Varadhan in [8], using the generalized duality techniques introduced by Landim and Yau [9] and Sethuraman, Varadhan and Yau [11]. Bernardin [1] proves regularity of the diffusion coefficient for nongradient reversible models under Bernoulli measures. A crucial step of the machinery for proving such regularities, consists in controlling the asymmetric part of the generator by the symmetric one. This is related to the so called sector conditions. We prove in this article a version of sector condition. The same proves applies to the case assumed by Komoriya in [3] to derive the hydrodynamic behavior of the mean zero exclusion process. In his work, he also assume regularity of the diffusion coefficient, main result of this work. Sector condition is the main point in the proof that the Hilbert space of fluctuations is the direct sum of gradients and local functions in the range of the generator.

To prove the sector condition, we use that every mean zero probability with finite support may be decomposed as a convex combination of loop probabilities. This idea was used by $\mathrm{Xu}$ in [14] when deducing the hydrodynamic behavior for the mean zero simple exclusion process and also by Varadhan in [13] when studying the evolution of the tagged particle in a mean zero exclusion process, in equilibrium. In Appendix A we give a simple proof of this decomposition.

This work is organized as follows. In Section 2 we introduce the notation and state the main theorem. In Section 3 we describe duality tools and describe several spaces and operators which appear in the dual representation. In Section 4 we state some results related to the sector conditions and give sufficient conditions for solving resolvent equation. In Section 5 we study the main properties of the Hilbert space of fluctuations which allow us to get in Section 6 a new expression for the diffusion coefficient. With this new expression, also in Section 6, we prove that the diffusion coefficient is a regular function.

\section{Notations and results}

\subsection{The model}

Fix a mean zero probability $p$ on $\mathbb{Z}_{*}^{d}=\mathbb{Z}^{d} \backslash\{0\}$, that vanishes outside a finite set and is irreducible. This last property means that $\{x: p(x)>0\}$ generates the whole group $\mathbb{Z}^{d}$. The generator of the simple exclusion process on $\mathbb{Z}^{d}$ associated to $p$ acts on local functions $f$ as

$$
(L f)(\eta)=\sum_{x, y \in \mathbb{Z}^{d}} p(y-x) \eta(x)\{1-\eta(y)\}\left[f\left(\eta^{x, y}\right)-f(\eta)\right]
$$

where $\eta^{x, y}$ stands for the configuration obtained from $\eta$ by exchanging the occupation variables $\eta(x), \eta(y)$ :

$$
\left(\eta^{x, y}\right)(z)= \begin{cases}\eta(z) & \text { if } z \neq x, y \\ \eta(x) & \text { if } z=y \\ \eta(y) & \text { if } z=x\end{cases}
$$


For $\alpha$ in $[0,1]$, denote by $v_{\alpha}$ the Bernoulli product measure on $\mathcal{X}=\{0,1\}^{\mathbb{Z}^{d}}$ with density $\alpha$. This one-parameter family of measures is stationary for the simple exclusion dynamics and in the symmetric case, $p(x)=p(-x)$, these measures are reversible. Expectation with respect to $v_{\alpha}$ is represented by $\langle\cdot\rangle_{\alpha}$ and the scalar product in $L^{2}\left(v_{\alpha}\right)$ by $\langle\cdot, \cdot\rangle_{\alpha} \cdot$

Denote by $s$ and $a$ the symmetric and the anti-symmetric parts of the probability $p$ :

$$
s(x)=(1 / 2)[p(x)+p(-x)], \quad a(x)=(1 / 2)[p(x)-p(-x)] .
$$

Let $L^{s}$ and $L^{a}$ be the symmetric and the anti-symmetric part of the generator $L$ in $L^{2}\left(v_{\alpha}\right)$, respectively. $L^{s}$ and $L^{a}$ are obtained replacing $p$ by $s, a$ in the definition of $L$. Also consider the probability $p^{*}(y)=p(-y)$ and let $L^{*}$ be the generator obtained replacing $p$ by $p^{*}$ in the definition of $L(2.1)$. Observe that $L^{*}$ is the adjoint operator of $L$ in $L^{2}\left(v_{\alpha}\right)$.

We will work on the torus. For a positive integer $N$, denote by $\mathbb{T}_{N}$ the torus with $N$ points $\mathbb{T}_{N}=\mathbb{Z} / N \mathbb{Z}$ and $\mathbb{T}_{N}^{d}=\left(\mathbb{T}_{N}\right)^{d}$. The continuous $d$-dimensional torus is denoted by $\mathbb{T}^{d}$ and is identified with $[0,1)^{d}$. Consider the exclusion process evolving in the torus $\mathbb{T}_{N}^{d}$. This is a Markov process on the state space $\chi_{N}=\{0,1\}^{\mathbb{T}_{N}^{d}}$, whose generator $L_{N}$ acts on a function $f$ as

$$
\left(L_{N} f\right)(\eta)=\sum_{x, y \in \mathbb{T}_{N}^{d}} p(y-x) \eta(x)\{1-\eta(y)\}\left[f\left(\eta^{x, y}\right)-f(\eta)\right] .
$$

\subsection{The hydrodynamic equation}

In order to deduce the hydrodynamic equation associated to this system, we look for the equation satisfied by the empirical measures. For a probability measure $\mu_{N}$ on $\chi_{N}$, denote by $\mathbb{P}_{\mu_{N}}$ the measure in $D\left([0, \infty), \chi_{N}\right)$ induced by the Markov process with generator $L_{N}$ speeded up by $N^{2}$, with initial distribution given by $\mu_{N}$. For each smooth function $H:[0, T] \times \mathbb{T}^{d} \rightarrow \mathbb{R}$, let $M^{H, N}(t)=M^{H}(t)$ be the martingale defined by:

$$
M^{H}(t)=\left\langle\pi_{t}^{N}, H_{t}\right\rangle-\left\langle\pi_{0}^{N}, H_{0}\right\rangle-\int_{0}^{t}\left(\partial_{s}+N^{2} L_{N}\right)\left\langle\pi_{s}^{N}, H_{s}\right\rangle d s,
$$

where $\pi_{t}^{N}=\pi^{N}\left(\eta_{t}\right)$ is the empirical measure associated to configuration $\eta_{t}$ and $\langle\pi, H\rangle$ is the integral of the function $H$ respect to the measure $\pi$. In general, $\pi^{N}(\eta)=N^{-d} \sum_{x \in \mathbb{T}_{N}^{d}} \eta(x) \delta_{x / N}$, where $\delta_{u}$ is the Dirac measure concentrated at $u$, and $\langle\pi, H\rangle=N^{-d} \sum_{x \in \mathbb{T}_{N}^{d}} \eta(x) H(x / N)$. Observe that

$$
L_{N} \eta(x)=\frac{1}{2} \sum_{y}\left\{W_{x-y, x}-W_{x, x+y}\right\},
$$

where the current $W_{x, x+y}$ between $x$ and $x+y$ in this model is given by

$$
W_{x, x+y}=\eta(x)[1-\eta(x+y)] p(y)-\eta(x+y)[1-\eta(x)] p(-y) .
$$

A spatial summation by parts and a second order approximation allow us to write

$$
\begin{aligned}
N^{2-d} \sum_{x \in \mathbb{T}_{N}^{d}} H_{S}(x / N) L_{N} \eta(x)= & \sum_{i} N^{1-d} \sum_{x \in \mathbb{T}_{N}^{d}} \partial_{u_{i}} H_{S}(x / N) \tau_{x} W_{i} \\
& +1 / 4 \sum_{i, j} N^{-d} \sum_{x \in \mathbb{T}_{N}^{d}} \partial_{u_{i}, u_{j}} H_{s}(x / N) \tau_{x} G_{i, j}+\mathrm{O}(1 / N),
\end{aligned}
$$

where

$$
W_{i}=1 / 2 \sum_{y} y_{i} W_{0, y}, \quad G_{i, j}=\sum_{y} y_{i} y_{j} W_{0, y}
$$


and $\left\{\tau_{x}, x \in \mathbb{Z}^{d}\right\}$ is the group of translations defined, initially, in the space of configuration by the formula

$$
\left(\tau_{x} \eta\right)(y)=\eta(x+y)
$$

and extended in a natural way to the space of functions with $\tau_{x} f(\eta)=f\left(\tau_{x} \eta\right)$. Observe that $E_{v_{\alpha}}\left[G_{i, j}\right]=0$ for every $\alpha$. This implies that the second term in the right hand side of (2.4) is negligible when deducing the hydrodynamic equation, thanks to a one block estimate argument. Then, it remains to replace $W_{i}$ by an object that allows us to do a second summation by parts. Following the nongradient method presented in Chapter 7 of [2], developed by Varadhan [12] and Quastel [10], we can prove that there exists a collection of functions $d_{i, j}:[0,1] \rightarrow \mathbb{R}$ such that

$$
\begin{aligned}
& \underset{\epsilon \rightarrow 0}{\limsup \limsup \mathbb{E}_{\mu_{N}}}\left[\mid \int_{0}^{t} N^{-d} \sum_{x \in \mathbb{T}_{N}^{d}} H(s, x / N)\right. \\
& \left.\quad \times N\left\{\tau_{x} W_{i}+\sum_{j=1}^{d}\left[d_{i, j}\left(\eta^{\epsilon N}\left(x+e_{j}\right)\right)-d_{i, j}\left(\eta^{\epsilon N}(x)\right)\right]\right\} d s \mid\right]=0 .
\end{aligned}
$$

This implies that the hydrodynamic behavior of this system is governed by the non-linear equation

$$
\partial_{t} \rho=\sum_{i, j=1}^{d} \partial_{u_{i}, u_{j}}^{2} d_{i, j}(\rho)=\sum_{i, j=1}^{d} \partial_{u_{i}}\left\{D_{i, j}(\rho) \partial_{u_{j}} \rho\right\}
$$

where $D_{i, j}(\alpha)=(d / d \alpha) d_{i, j}(\alpha)$. The following goal is to give an explicit form for the diffusion coefficients $D_{i, j}$, appearing in the previous equation.

\subsection{The diffusion coefficient and main result}

Let introduce a semi-norm in $\mathcal{C}_{0}$, the space of local functions with mean zero with respect to all grand canonical measures $v_{\alpha}$. Denote $\chi(\alpha)=\alpha(1-\alpha)$. For $h$ in $\mathcal{C}_{0}$, consider

$$
\langle h\rangle_{\alpha}=\sup _{a \in \mathbb{R}^{d}}\left\{2 \sum_{i=1}^{d} a_{i}\left\langle\langle h\rangle_{\alpha, i}-\frac{1}{2} \chi(\alpha) \sum_{v} s(v)\left(\sum_{i=1}^{d} a_{i} v_{i}\right)^{2}\right\}+\sup _{g \in \mathcal{C}_{0}}\left\{2\langle h, g\rangle_{\alpha, 0}-\left\langle\left\langle-L^{s} g, g\right\rangle_{\alpha, 0}\right\},\right.\right.
$$

where

$$
\langle h\rangle_{\alpha, i}=\sum_{x \in \mathbb{Z}^{d}}\left\langle x_{i} \eta(x) h\right\rangle_{\alpha}, \quad\langle h, g\rangle_{\alpha, 0}=\sum_{x \in \mathbb{Z}^{d}}\left\langle\tau_{x} h, g\right\rangle_{\alpha}
$$

and $\left\{\tau_{x}, x \in \mathbb{Z}^{d}\right\}$ is the group of translations defined in (2.5).

In Lemma 5.2 we prove that $\langle h\rangle_{\alpha}$ is finite for any $h \in \mathcal{C}_{0}$. Also, it may be proved that $\langle\cdot\rangle_{\alpha}$ verifies the parallelogram identity. Then, there exists a semi-inner product on $\mathcal{C}_{0}$ associated to the semi-norm. Denote by $H_{\alpha}$ Hilbert space induced by $\left\langle\langle\cdot, \cdot\rangle_{\alpha}\right.$ on $\mathcal{C}_{0}$. The techniques developed to study nongradient systems (see Section 7 in [2]), shows that the matrix $D=\left\{D_{i, j}(\alpha)\right\}_{1 \leqslant i, j \leqslant d}$ is such that

$$
W_{i}+\sum_{j} D_{i, j}(\alpha)\left[\eta\left(e_{j}\right)-\eta(0)\right] \in \overline{L \mathcal{C}_{0}}
$$

in $H_{\alpha}$, for $0<\alpha<1$, where $W_{i}$ are the functions defined in (2.3). In other words, $D_{i, j}(\alpha)$ are characterized by the following property:

$$
\inf _{u \in \mathcal{C}_{0}}\left\langle\left\langle W_{i}+\sum_{j} D_{i, j}(\alpha)\left[\eta\left(e_{j}\right)-\eta(0)\right]-L u \|_{\alpha}=0 .\right.\right.
$$


In the symmetric case, we have a gradient model that allows to perform a second summation by parts when deducing the hydrodynamic equation, given by

$$
\partial_{t} \rho=\sum_{i, j=1}^{d} \partial_{u_{i}}\left\{\frac{1}{2} \sigma_{i, j} \partial_{u_{j}} \rho\right\},
$$

where, since the probability $p$ is irreducible, $\sigma=\left\{\sigma_{i, j}\right\}$ is the strictly positive defined matrix given by

$$
\sigma_{i, j}=\sum_{y} s(y) y_{i} y_{j}
$$

As in Corollary 6.2 in [5], we can prove that

$$
\begin{aligned}
\beta^{*} D^{s}(\alpha) \beta-\frac{1}{2} \beta^{*} \sigma \beta= & \frac{1}{\chi(\alpha)} \sup _{g \in \mathcal{C}_{0}}\left\{\sum_{j}(\sigma \beta)_{j}\left\|\eta\left(e_{j}\right)-\eta(0), L^{*} g\right\|_{\alpha}\right. \\
& +\frac{1}{2 \chi(\alpha)} \sum_{k, i} \sigma_{k, i}\left\|\eta\left(e_{i}\right)-\eta(0), L^{*} g\right\|_{\alpha}\left\|\eta\left(e_{k}\right)-\eta(0), L^{*} g\right\|_{\alpha}-\left\langle\left\langle L^{*} g, L^{*} g\right\rangle_{\alpha}\right\},
\end{aligned}
$$

where $D^{s}(\alpha)$ is the symmetric part of the matrix $D(\alpha)$, given by

$$
D^{s}(\alpha)_{i, j}=\frac{1}{2}\left(D(\alpha)_{i, j}+D(\alpha)_{j, i}\right)
$$

The main result of this work is the following.

Theorem 2.1. The function $D_{i, j}(\alpha)$ is $C^{\infty}$ on $[0,1]$, for $1 \leqslant i, j \leqslant d$.

In order to prove this result, we need to find an appropriate expression for $D_{i, j}(\alpha)$. This is done in Section 6 , where we study deeply the structure of the Hilbert space $H_{\alpha}$. Observe that the first term in (2.7) is easy to compute and is a smooth function of $\alpha$. The next sections are consecrated to deal with the second term of (2.7).

\section{Duality}

Considering the second line in formula (2.7), we examine in this section the action of the symmetric part $L^{s}$ of the generator on the space of local functions endowed with the scalar product $\langle\cdot \cdot, \cdot\rangle_{\alpha, 0}$. Some notations and computations of this section are taken from [7]. Fix, once for all, a density $\alpha$ in $(0,1)$. All expectations in this section are taken with respect to $v_{\alpha}$ and we omit all subscripts.

\subsection{The dual space}

For each $n \geqslant 0$, denote by $\mathcal{E}_{n}$ the subsets of $\mathbb{Z}^{d}$ with $n$ points and let $\mathcal{E}=\bigcup_{n \geqslant 0} \mathcal{E}_{n}$ be the class of finite subsets of $\mathbb{Z}^{d}$. For each $A$ in $\mathcal{E}$, let $\Psi_{A}$ be the local function

$$
\Psi_{A}=\prod_{x \in A} \frac{\eta(x)-\alpha}{\sqrt{\chi(\alpha)}},
$$

where $\chi(\alpha)=\alpha(1-\alpha)$. By convention, $\Psi_{\phi}=1$. It is easy to check that $\left\{\Psi_{A}, A \in \mathcal{E}\right\}$ is an orthonormal basis of $L^{2}\left(v_{\alpha}\right)$. For each $n \geqslant 0$, denote by $\mathcal{G}_{n}$ the subspace of $L^{2}\left(v_{\alpha}\right)$ generated by $\left\{\Psi_{A}, A \in \mathcal{E}_{n}\right\}$, so that $L^{2}\left(v_{\alpha}\right)=$ 
$\bigoplus_{n \geqslant 0} \mathcal{G}_{n}$. Functions in $\mathcal{G}_{n}$ are said to have degree $n$. We use $\pi_{n}$ to denote the projection operator from $L^{2}\left(v_{\alpha}\right)$ to the subspace $\mathcal{G}_{n}$. Then, given a function $f$ in $L^{2}\left(v_{\alpha}\right)$, we may write

$$
f=\sum_{n \geqslant 0} \pi_{n} f=\sum_{n \geqslant 0} \sum_{A \in \mathcal{E}_{n}} \mathfrak{f}(A) \Psi_{A} .
$$

Note that the coefficients $\mathfrak{f}(A)$ depend not only on $f$ but also on the density $\alpha: \mathfrak{f}(A)=\mathfrak{f}(A, \alpha)$. If $f$ is a local function, $\mathfrak{f}: \mathcal{E} \rightarrow \mathbb{R}$ is a function of finite support. Denote by $\mathcal{C}$ the space of local functions and recall that $\mathcal{C}_{0}$ is the set of local functions that have mean zero with respect to all grand canonical measure $v_{\beta}$. We have a simple characterization of $\mathcal{C}_{0}$ functions in terms of their Fourier coefficients:

$$
f \in \mathcal{C}_{0} \quad \Leftrightarrow \quad \sum_{A \in \mathcal{E}_{n}} \mathfrak{f}(A, \beta)=0 \quad \forall n \geqslant 0, \forall \beta .
$$

For local functions $u, v$ in $\mathcal{C}_{0}$, define the scalar product $\left\langle\langle\cdot, \cdot\rangle\right.$ (previously noted by $\left\langle\langle\cdot, \cdot\rangle_{\alpha, 0}\right.$ ) by

$$
\langle u, v\rangle=\sum_{x \in \mathbb{Z}^{d}}\left\langle\tau_{x} u, v\right\rangle,
$$

where $\left\{\tau_{x}, x \in \mathbb{Z}^{d}\right\}$ is the group of translations. Since $\left\langle u-\tau_{x} u, v\right\rangle=0$ for all $x$ in $\mathbb{Z}^{d}$, this scalar product is only semi-definite positive (formula (3.6) below guarantees $\langle u, u\rangle \geqslant 0$ ). Denote by $L_{\langle\cdot, \cdot,\rangle}^{2}\left(v_{\alpha}\right)$ the Hilbert space generated by the local functions in $\mathcal{C}_{0}$ and the inner product $\langle\cdot \cdot, \cdot\rangle$. The scalar product of two local functions $u$, $v$ can be written in terms of the Fourier coefficients of $u, v$ through a simple formula. To this end, fix two local functions $u, v$ and write them in the basis $\left\{\Psi_{A}, A \in \mathcal{E}\right\}$ :

$$
u=\sum_{A \in \mathcal{E}} \mathfrak{u}(A) \Psi_{A}, \quad v=\sum_{A \in \mathcal{E}} \mathfrak{v}(A) \Psi_{A} .
$$

An elementary computation shows that

$$
\langle u, v\rangle=\sum_{x \in \mathbb{Z}^{d}} \sum_{n \geqslant 1} \sum_{A \in \mathcal{E}_{n}} \mathfrak{u}(A) \mathfrak{v}(A+x) .
$$

In this formula, $B+z$ is the set $\{x+z ; x \in B\}$. The summation starts from $n=1$ because we are working with functions in $\mathcal{C}_{0}$.

We say that two finite subsets $A, B$ of $\mathbb{Z}^{d}$ are equivalent if one is the translation of the other. This equivalence relation is denoted by $\sim$ so that $A \sim B$ if $A=B+x$ for some $x$ in $\mathbb{Z}^{d}$. Let $\tilde{\mathcal{E}}_{n}$ be the quotient of $\mathcal{E}_{n}$ with respect to this equivalence relation: $\tilde{\mathcal{E}}_{n}=\mathcal{E}_{n} / \sim, \tilde{\mathcal{E}}=\mathcal{E} / \sim$. For any summable function $\mathfrak{f}: \mathcal{E} \rightarrow \mathbb{R}$,

$$
\sum_{A \in \mathcal{E}} \mathfrak{f}(A)=\sum_{A \in \tilde{\mathcal{E}} z \in \mathbb{Z}^{d}} \sum_{\mathfrak{f}}(A+z) .
$$

In particular, for two local functions $u, v$,

$$
\langle u, v\rangle=\sum_{x, z \in \mathbb{Z}^{d}} \sum_{n \geqslant 1} \sum_{A \in \tilde{\mathcal{E}}_{n}} \mathfrak{u}(A+z) \mathfrak{v}(A+x+z)=\sum_{n \geqslant 1} \sum_{A \in \tilde{\mathcal{E}}_{n}} \tilde{\mathfrak{u}}(A) \tilde{\mathfrak{v}}(A),
$$

where, for a finite set $A$ and a summable function $\mathfrak{u}: \mathcal{E} \rightarrow \mathbb{R}$,

$$
\tilde{\mathfrak{u}}(A)=\sum_{z \in \mathbb{Z}^{d}} \mathfrak{u}(A+z) .
$$

We say that a function $\mathfrak{f}: \mathcal{E} \rightarrow \mathbb{R}$ is translation invariant if $\mathfrak{f}(A+x)=\mathfrak{f}(A)$ for all sets $A$ in $\mathcal{E}$ and all sites $x$ of $\mathbb{Z}^{d}$. Of course, the functions $\tilde{\mathfrak{u}}$ are translation invariant. Fix a subset $A$ of $\mathbb{Z}^{d}$ with $n$ points. There are $n$ sets in the class of equivalence of $A$ that contain the origin. Therefore

$$
\sum_{A \in \tilde{\mathcal{E}}_{n}} \mathfrak{f}(A)=\frac{1}{n} \sum_{\substack{A \in \mathcal{E}_{n} \\ A \ni 0}} \mathfrak{f}(A)
$$


if $\mathfrak{f}(A)=\mathfrak{f}(A+x)$ for all $A$, for all $x$. Let $\mathcal{E}_{*}$ be the class of all finite subsets of $\mathbb{Z}_{*}^{d}=\mathbb{Z}^{d} \backslash\{0\}$ and let $\mathcal{E}_{*, n}$ be the class of all subsets of $\mathbb{Z}_{*}^{d}$ with $n$ points. Then, we may write

$$
\langle u, v\rangle=\sum_{n \geqslant 1} \frac{1}{n} \sum_{\substack{A \in \mathcal{E}_{n} \\ A \ni 0}} \tilde{\mathfrak{u}}(A) \tilde{\mathfrak{v}}(A)=\sum_{n \geqslant 0} \frac{1}{n+1} \sum_{A \in \mathcal{E}_{*, n}} \tilde{\mathfrak{u}}(A \cup\{0\}) \tilde{\mathfrak{v}}(A \cup\{0\}) .
$$

Summarizing, for a finitely supported function $\mathfrak{f}: \mathcal{E} \rightarrow \mathbb{R}$, define $\mathfrak{T f}: \mathcal{E}_{*} \rightarrow \mathbb{R}$ by

$$
(\mathfrak{T} \mathfrak{f})(A)=\tilde{\mathfrak{f}}(A \cup\{0\})=\sum_{z \in \mathbb{Z}^{d}} \mathfrak{f}([A \cup\{0\}]+z),
$$

then we have that

$$
\langle u, v\rangle=\sum_{n \geqslant 0} \frac{1}{n+1} \sum_{A \in \mathcal{E}_{*, n}} \mathfrak{T} \mathfrak{u}(A) \mathfrak{T} \mathfrak{v}(A) .
$$

To state some properties of the transformation $\mathfrak{T}$, we need some notation. For a subset $A$ of $\mathbb{Z}_{*}^{d}$ and $z$ in $\mathbb{Z}_{*}^{d}, S_{z} A$ is the set defined by

$$
S_{z} A= \begin{cases}A-z & \text { if } z \notin A, \\ {[(A-z) \backslash\{0\}] \cup\{-z\}} & \text { if } z \in A .\end{cases}
$$

Therefore, to obtain $S_{z} A$ from $A$ when $z$ belongs to $A$, we first translate $A$ by $-z$, getting a new set which contains the origin, and we then remove the origin and add site $-z$.

\section{Remark 3.1.}

(a) Since $f$ belongs to $\mathcal{C}_{0}$, from (3.2) we get that

$$
\mathfrak{T} \mathfrak{f}(\phi)=\sum_{z \in \mathbb{Z}^{d}} \mathfrak{f}(\{z\})=0 .
$$

(b) Not any function $\mathfrak{f}_{*}: \mathcal{E}_{*} \rightarrow \mathbb{R}$ is the image by $\mathfrak{T}$ of some function $\mathfrak{f}: \mathcal{E} \rightarrow \mathbb{R}$ since

$$
(\mathfrak{T} \mathfrak{f})(A)=(\mathfrak{T f})\left(S_{z} A\right)
$$

for all $z$ in $A$.

(c) Let $\mathfrak{f}_{*}: \mathcal{E}_{*} \rightarrow \mathbb{R}$ be a finitely supported function with $\mathfrak{f}_{*}(\phi)=0$ and satisfying (3.8): $\mathfrak{f}_{*}(A)=\mathfrak{f}_{*}\left(S_{z} A\right)$ for all $z$ in $A$. Define $\mathfrak{f}: \mathcal{E} \rightarrow \mathbb{R}$ by

$$
\mathfrak{f}(B)= \begin{cases}|B|^{-1} \mathfrak{f}_{*}(B \backslash\{0\}) & \text { if } B \ni 0, \\ 0 & \text { otherwise. }\end{cases}
$$

An elementary computations shows that $\mathfrak{T} \mathfrak{f}=\mathfrak{f}_{*}$. This choice, which is not unique, makes $\mathfrak{f}$ vanish on $\mathcal{E}_{1}$.

(d) The operation that transforms $\mathfrak{f}$ in $\mathfrak{T} \mathfrak{f}$ reduces by one the degree of a function.

To keep notation simple, most of the times, real functions on $\mathcal{E}$ or on $\mathcal{E}_{*}$ are indistinctively denoted by the symbols $\mathfrak{f}, \mathfrak{g}, \mathfrak{u}, \mathfrak{v}$.

\subsection{Some Hilbert spaces}

For $n \geqslant 0$, let

$$
L^{2}\left(\mathcal{E}_{*, n}\right)=\left\{\mathfrak{f}: \mathcal{E}_{*, n} \rightarrow \mathbb{R}: \sum_{A \in \mathcal{E}_{*, n}} \mathfrak{f}^{2}(A)<\infty\right\}
$$


and for $\mathfrak{f}, \mathfrak{g}$ in $L^{2}\left(\mathcal{E}_{*, n}\right)$, define $\langle\mathfrak{f}, \mathfrak{g}\rangle=\sum_{A \in \mathcal{E}_{*, n}} \mathfrak{f}(A) \mathfrak{g}(A)$ for every $n$. We put $\|\mathfrak{f}\|^{2}=\langle\mathfrak{f}, \mathfrak{f}\rangle$ whenever $\mathfrak{f} \in L^{2}\left(\mathcal{E}_{*, n}\right)$. Define, analogously, $L^{2}\left(\mathcal{E}_{n}\right)$. For $n \geqslant 0$ consider the following spaces:

$$
\begin{aligned}
& \mathcal{F}_{n}=\left\{\mathfrak{f}: \mathcal{E}_{n} \rightarrow \mathbb{R}: \mathfrak{f}(A) \neq 0 \text { for a finite number of sets } A \text { and } \sum_{A \in \mathcal{E}_{n}} \mathfrak{f}(A)=0\right\}, \\
& \mathcal{F}_{*, n}= \begin{cases}\left.\mathfrak{f}: \mathcal{E}_{*, n} \rightarrow \mathbb{R}: \begin{array}{l}
\mathfrak{f}(A) \neq 0 \text { for a finite number of sets } A, \\
\sum_{A \in \mathcal{E}_{*, n}} \mathfrak{f}(A)=0 \text { and } \mathfrak{f}\left(S_{z} B\right)=\mathfrak{f}(B) \\
\text { for all } B \in \mathcal{E}_{*, n}, \text { for all } z \in B
\end{array}\right\} .\end{cases}
\end{aligned}
$$

From $\sum_{A \in \mathcal{E}_{*, 0}} \mathfrak{f}(A)=\mathfrak{f}(\emptyset)=0$, we get that $\mathcal{F}_{*, 0}=\{0\}$.

Observe that the operator $\mathfrak{T}$, defined by formula (3.5), maps $\mathcal{F}_{n}$ to $\mathcal{F}_{*, n-1}$. A function $\mathfrak{f} \in \mathcal{F}_{n}$ or $\mathfrak{f} \in \mathcal{F}_{*, n}$ is called a finite supported function of degree $n$. Put $\mathcal{I}_{n}$ and $\mathcal{I}_{*, n}$ for the closure of $\mathcal{F}_{n}$ and $\mathcal{F}_{*, n}$ as subspaces of $L^{2}\left(\mathcal{E}_{n}\right)$ and $L^{2}\left(\mathcal{E}_{*, n}\right)$, respectively. For $\mathfrak{f}: \mathcal{E}_{*} \rightarrow \mathbb{R}$ define the projection $\pi_{n}$ by

$$
\left(\pi_{n} \mathfrak{f}\right)(A)= \begin{cases}\mathfrak{f}(A) & \text { if }|A|=n, \\ 0 & \text { otherwise. }\end{cases}
$$

Let

$$
\mathcal{I}_{*}=\left\{\mathfrak{f}: \mathcal{E}_{*} \rightarrow \mathbb{R}: \pi_{n} \mathfrak{f} \in \mathcal{I}_{*, n} \forall n \geqslant 0\right\} .
$$

Given a local function in $\mathcal{C}_{0}$, take its Fourier coefficients in $L^{2}\left(v_{\alpha}\right)$ and apply the operator $\mathfrak{T}$ to the Fourier coefficients. The image of this transformation belongs to the space of finite supported functions given by

$$
\mathcal{F}_{*}=\left\{\mathfrak{f}: \mathcal{E}_{*} \rightarrow \mathbb{R}: \begin{array}{l}
\pi_{n}(\mathfrak{f})=0 \text { for all } n \geqslant n_{0}, \text { for some } n_{0}, \\
\text { and } \pi_{n}(\mathfrak{f}) \in \mathcal{F}_{*, n} \text { for all } n \geqslant 0
\end{array}\right\} .
$$

Consider the inner product $\langle\cdot, \cdot\rangle_{0, k}$ in the space $\mathcal{F}_{*}$, given by:

$$
\langle\mathfrak{f}, \mathfrak{g}\rangle_{0, k}=\sum_{n \geqslant 0}(n+1)^{2 k-1}\left\langle\pi_{n} \mathfrak{f}, \pi_{n} \mathfrak{g}\right\rangle
$$

so that

$$
\|\mathfrak{f}\|_{0, k}^{2}=\sum_{n \geqslant 0}(n+1)^{2 k-1}\left\langle\pi_{n} \mathfrak{f}, \pi_{n} \mathfrak{f}\right\rangle
$$

The term corresponding to $n=0$ in each of the previous expressions is equal to zero. Let $\mathbb{I}_{*}^{k}$ be the Hilbert space induced by the inner product $\langle\cdot, \cdot\rangle_{0, k}$ and $\mathcal{F}_{*}$. Observe that we have the following embeddings:

$$
\mathbb{I}_{*}^{0} \hookleftarrow \mathbb{I}_{*}^{1} \cdots \hookleftarrow \mathbb{I}_{*}^{s} \hookleftarrow \mathbb{I}_{*}^{s+1} \cdots
$$

An explicit way to construct the spaces $\mathbb{I}_{*}^{k}$ is adding the Hilbert spaces $\mathcal{I}_{*, n}$ weighted by $(n+1)^{2 k-1}$ :

$$
\mathbb{I}_{*}^{k}=\left\{\mathfrak{f} \in \mathcal{I}_{*}: \sum_{n}(n+1)^{2 k-1}\left\|\pi_{n} \mathfrak{f}\right\|^{2}<\infty\right\} .
$$

With this notation, for local functions $f$ and $g$, in view of (3.6), we have that

$$
\langle f, g\rangle=\left\langle\langle\mathfrak{T} \mathfrak{f}, \mathfrak{T} \mathfrak{g}\rangle_{0,0},\right.
$$

where $\mathfrak{f}$ and $\mathfrak{g}$ are the Fourier coefficients of $f$ and $g$, respectively.

We now examine the action of the symmetric part of the generator $L$ on the basis $\left\{\Psi_{A}, A \in \mathcal{E}\right\}$ (see diagram (3.31) below as reference). Fix a function $u \in \mathcal{C}_{0}$ and denote by $\mathfrak{u}$ its Fourier coefficients. A straightforward computation shows that

$$
L^{s} u=\sum_{A \in \mathcal{E}}\left(\mathcal{L}_{s} \mathfrak{u}\right)(A) \Psi_{A}
$$


where $\mathcal{L}_{S}$ is the generator of finite symmetric random walks evolving with exclusion on $\mathbb{Z}^{d}$ :

$$
\left(\mathcal{L}_{s} \mathfrak{u}\right)(A)=(1 / 2) \sum_{x, y \in \mathbb{Z}^{d}} s(y-x)\left[\mathfrak{u}\left(A_{x, y}\right)-\mathfrak{u}(A)\right]
$$

and $A_{x, y}$ is the set defined by

$$
A_{x, y}= \begin{cases}(A \backslash\{x\}) \cup\{y\} & \text { if } x \in A, y \notin A, \\ (A \backslash\{y\}) \cup\{x\} & \text { if } y \in A, x \notin A, \\ A & \text { otherwise. }\end{cases}
$$

Furthermore, an elementary computation, based on the fact that

$$
\sum_{z \in \mathbb{Z}^{d}} \mathfrak{f}([B \cup\{y\}]+z)=\mathfrak{T} \mathfrak{f}\left(S_{y} B\right)
$$

for all subsets $B$ of $\mathbb{Z}_{*}^{d}$, sites $y$ not in $B$ and finitely supported functions $\mathfrak{f}: \mathcal{E} \rightarrow \mathbb{R}$, shows that for every set $B$ in $\mathcal{E}_{*}$

$$
\mathfrak{T} \mathcal{L}_{s} \mathfrak{u}(B)=\mathfrak{L}_{s} \mathfrak{T} \mathfrak{u}(B),
$$

where

$$
\left(\mathfrak{L}_{s} \mathfrak{v}\right)(B)=(1 / 2) \sum_{x, y \in \mathbb{Z}_{*}^{d}} s(y-x)\left[\mathfrak{v}\left(B_{x, y}\right)-\mathfrak{v}(B)\right]+\sum_{y \notin B, y \neq 0} s(y)\left[\mathfrak{v}\left(S_{y} B\right)-\mathfrak{v}(B)\right] .
$$

We are now in a position to define the Hilbert space induced by the local functions in $\mathcal{C}_{0}$, the symmetric part of the generator $L$ and the scalar product $\langle\cdot, \cdot\rangle\rangle$. For two local functions $u, v$ in $\mathcal{C}_{0}$, let

$$
\langle u, v\rangle_{1}=\left\langle\left\langle u,\left(-L^{s}\right) v\right\rangle\right.
$$

and let $\left.H_{1}=H_{1}\left(\mathcal{C}_{0}, L^{S},\langle\cdot, \cdot\rangle\right)\right)$ be the Hilbert space generated by mean zero local functions $f$ and the inner product $\langle\cdot, \cdot\rangle_{1}$. By (3.14), (3.6) and (3.17) the previous scalar product is equal to

$$
-\sum_{n \geqslant 0} \frac{1}{n+1} \sum_{A \in \mathcal{E}_{*, n}} \mathfrak{T} \mathfrak{u}(A) \mathfrak{T} \mathcal{L}_{s} \mathfrak{v}(A)=-\sum_{n \geqslant 0} \frac{1}{n+1} \sum_{A \in \mathcal{E}_{*, n}} \mathfrak{T} \mathfrak{u}(A)\left(\mathfrak{L}_{s} \mathfrak{T} \mathfrak{v}\right)(A)=\sum_{n \geqslant 0} \frac{1}{n+1}\left\langle\pi_{n} \mathfrak{T} \mathfrak{u},\left(-\mathfrak{L}_{s}\right) \pi_{n} \mathfrak{T} \mathfrak{v}\right\rangle
$$

because $\mathfrak{L}_{s}$ keeps the degree of the functions mapping $L^{2}\left(\mathcal{E}_{*, n}\right)$ in itself.

Now, for each $n \geqslant 0$, denote by $\langle\cdot, \cdot\rangle_{1}$ the scalar product on $\mathcal{F}_{*, n}$ defined by

$$
\langle\mathfrak{f}, \mathfrak{g}\rangle_{1}=\left\langle\mathfrak{f},\left(-\mathfrak{L}_{s}\right) \mathfrak{g}\right\rangle
$$

and denote by $\mathfrak{H}_{1}\left(\mathcal{F}_{*, n}\right)$ the Hilbert space induced by the scalar product $\langle\cdot, \cdot\rangle_{1}$ on $\mathcal{F}_{*, n}$. The associated norm is denoted by $\|\mathfrak{f}\|_{1}^{2}=\left\langle\mathfrak{f},\left(-\mathfrak{L}_{s}\right) \mathfrak{f}\right\rangle$. Furthermore, for an integer $k \geqslant 0$, denote by $\mathfrak{H}_{1, k}=\mathfrak{H}_{1}\left(\mathcal{F}_{*}, \mathfrak{L}_{s}, k\right)$ the Hilbert space induced by the finitely supported functions $\mathfrak{f}, \mathfrak{g} \in \mathcal{F}_{*}$ with scalar product

$$
\langle\langle\mathfrak{f}, \mathfrak{g}\rangle\rangle_{1, k}=\left\langle\mathfrak{f},\left(-\mathfrak{L}_{s}\right) \mathfrak{g}\right\rangle_{0, k}=\sum_{n \geqslant 0}(n+1)^{2 k-1}\left\langle\pi_{n} \mathfrak{f},\left(-\mathfrak{L}_{s}\right) \pi_{n} \mathfrak{g}\right\rangle .
$$

The associated norm is denoted by $\|\cdot\|_{1, k}$ so that

$$
\|\mathfrak{f}\|_{1, k}^{2}=\left\langle\langle\mathfrak{f}, \mathfrak{f}\rangle_{1, k} .\right.
$$

It follows from the previous notation that

$$
\|\mathfrak{f}\|_{1, k}^{2}=\sum_{n \geqslant 0}(n+1)^{2 k-1}\left\|\pi_{n} \mathfrak{f}\right\|_{1}^{2} .
$$

Observe that for every local function $u, v \in \mathcal{C}_{0}$,

$$
\langle u, v\rangle_{1}=\left\langle\langle\mathfrak{T} \mathfrak{u}, \mathfrak{T} \mathfrak{v}\rangle_{1,0},\right.
$$


where $\mathfrak{u}$ and $\mathfrak{v}$ are the Fourier coefficients of $u$ and $v$, respectively.

To introduce $H_{-1}$, the dual space of $H_{1}$, consider the functions $u \in \mathcal{C}_{0}$ that define a bounded operator respect to the $\|\cdot\|_{1}$ norm and the inner product $\left.\langle\cdot, \cdot\rangle\right\rangle$ : it means that there exists a constant $C$ with

$$
|\langle u, v\rangle| \leqslant C\|v\|_{1} \quad \text { for all } v \in \mathcal{C}_{0} .
$$

The smallest $C$ satisfying the previous condition is denoted by $\|u\|_{-1}$ and satisfies the following variational formula:

$$
\|u\|_{-1}^{2}=\sup _{v}\{2\langle u, v\rangle\rangle-\left\langle\langle v, v\rangle_{1}\right\}
$$

where the supremum is taken over all local functions $v$ in $\mathcal{C}_{0}$. Denote by $H_{-1}=H_{-1}\left(\mathcal{C}_{0}, L^{s},\langle\cdot, \cdot\rangle\right)$ the Hilbert space generated by the local functions and the semi-norm $\|\cdot\|_{-1}$.

Since $L^{s}$ keeps the degree of a function and since the spaces $\mathcal{G}_{n}$ are orthogonal, for local functions of degree $n$, we may restrict the supremum to local functions of the same degree, so that

$$
\|f\|_{-1}^{2}=\sum_{n \geqslant 1}\left\|\pi_{n} f\right\|_{-1}^{2} .
$$

In the same way, for an integer $n \geqslant 1$ and a finitely supported function $\mathfrak{u} \in \mathcal{F}_{*, n}$, let

$$
\|\mathfrak{u}\|_{-1}^{2}=\sup _{\mathfrak{v}}\left\{2\langle\mathfrak{u}, \mathfrak{v}\rangle-\langle\mathfrak{v}, \mathfrak{v}\rangle_{1}\right\}
$$

where the supremum is carried over all finitely supported functions $\mathfrak{v} \in \mathcal{F}_{*, n}$. Observe that, as when defining $H_{-1}$, we have that $\|\mathfrak{u}\|_{-1}$ is the smallest constant $C>0$ verifying

$$
|\langle\mathfrak{v}, \mathfrak{u}\rangle| \leqslant C\|\mathfrak{v}\|_{1} \quad \text { for all } \mathfrak{v} \in \mathcal{F}_{*, n} .
$$

Denote by $\mathfrak{H}_{-1}=\mathfrak{H}_{-1}\left(\mathcal{F}_{*, n}\right)$ the Hilbert space induced by the finitely supported functions $\mathfrak{u} \in \mathcal{F}_{*, n}$ and the semi-norm $\|\cdot\|_{-1}$.

For a integer $k \geqslant 0$, define the $\|\cdot\|_{-1, k}$ norm of a finite supported function $\mathfrak{u} \in \mathcal{F}_{*}$ by

$$
\|\mathfrak{u}\|_{-1, k}^{2}=\sup _{\mathfrak{v}}\left\{2\langle\mathfrak{u}, \mathfrak{v}\rangle_{0, k}-\left\langle\left\langle\mathfrak{v},\left(-\mathfrak{L}_{s}\right) \mathfrak{v}\right\rangle_{0, k}\right\}\right.
$$

where the supremum is carried over all finitely supported functions $\mathfrak{v} \in \mathcal{F}_{*}$. Denote by $\mathfrak{H}_{-1, k}=\mathfrak{H}_{-1}\left(\mathcal{F}_{*}, \mathfrak{L}_{s}, k\right)$ the Hilbert space induced by this semi-norm and the space of finite supported functions. Here again, since $\mathfrak{L}_{s}$ does not change the degrees of a function, for every finitely supported $\mathfrak{u} \in \mathcal{F}_{*}$,

$$
\|\mathfrak{u}\|_{-1, k}^{2}=\sum_{n \geqslant 1}(n+1)^{2 k-1}\left\|\pi_{n} \mathfrak{u}\right\|_{-1}^{2}
$$

and for any local function $u \in \mathcal{C}_{0}$, since $\pi_{0} u=0$, we get that

$$
\|u\|_{-1}=\|\mathfrak{T} \mathfrak{u}\|_{-1,0},
$$

where $\mathfrak{u}$ denotes the Fourier coefficient of $u$.

We end this subsection summarizing the different norms recently defined. In $\mathcal{C}_{0}$ we have

$$
\begin{aligned}
& \langle u, v\rangle=\sum_{x \in \mathbb{Z}^{d}}\left\langle\tau_{x} u, v\right\rangle, \quad\|u\|_{1}^{2}=\left\langle u,-L_{s} u\right\rangle, \\
& \|u\|_{-1}^{2}=\sup _{v \in \mathcal{C}_{0}}\left\{2\langle u, v\rangle-\left\langle\langle v, v\rangle_{1}\right\} .\right.
\end{aligned}
$$


In $\mathcal{F}_{*, n}$

$$
\begin{aligned}
\langle\mathfrak{u}, \mathfrak{v}\rangle & =\sum_{A \in \mathcal{E}_{*, n}} \mathfrak{u}(A) \mathfrak{v}(A), \quad\|\mathfrak{u}\|_{1}^{2}=\left\langle\mathfrak{u},-\mathfrak{L}_{s} \mathfrak{u}\right\rangle, \\
\|\mathfrak{u}\|_{-1}^{2} & =\sup _{\mathfrak{v} \in \mathcal{F}_{*, n}}\left\{2\langle\mathfrak{u}, \mathfrak{v}\rangle-\langle\mathfrak{v}, \mathfrak{v}\rangle_{1}\right\} .
\end{aligned}
$$

Adding the respective norms with appropriate weights, in $\mathcal{F}_{*}$ we get that

$$
\begin{aligned}
\langle u \mathfrak{u}, \mathfrak{v}\rangle_{0, k} & =\sum_{n}(n+1)^{2 k-1}\left\langle\pi_{n} \mathfrak{u}, \pi_{n} \mathfrak{v}\right\rangle, \\
\langle\mathfrak{u}, \mathfrak{v}\rangle_{1, k} & =\sum_{n}(n+1)^{2 k-1}\left\langle\pi_{n} \mathfrak{u}, \pi_{n} \mathfrak{v}\right\rangle_{1}, \\
\|\mathfrak{u}\|_{-1, k}^{2} & =\sum_{n}(n+1)^{2 k-1}\left\|\pi_{n} \mathfrak{u}\right\|_{-1}^{2} .
\end{aligned}
$$

\subsection{The Fourier coefficients of the generator $L$}

We conclude this section deriving explicit expressions for the generator $L$ on the basis $\left\{\Psi_{A}, A \subset \mathbb{Z}^{d}\right\}$. A long and simple computation gives the following dual representation: for every local function $u=\sum_{A \in \mathcal{E}} \mathfrak{u}(A) \Psi_{A}$,

$$
L u=\sum_{A \in \mathcal{E}}\left(\mathcal{L}_{\alpha} \mathfrak{u}\right)(A) \Psi_{A}, \quad L^{a} u=\sum_{A \in \mathcal{E}}\left(\mathcal{L}_{a, \alpha} \mathfrak{u}\right)(A) \Psi_{A},
$$

where $\mathcal{L}_{\alpha}=\mathcal{L}_{s}+(1-2 \alpha) \mathcal{L}_{d}+\sqrt{\chi(\alpha)}\left(\mathcal{L}_{+}+\mathcal{L}_{-}\right), \mathcal{L}_{a, \alpha}=\mathcal{L}_{\alpha}-\mathcal{L}_{s}$

$$
\begin{aligned}
& \left(\mathcal{L}_{d} \mathfrak{u}\right)(A)=\sum_{x \in A, y \notin A} a(y-x)\left\{\mathfrak{u}\left(A_{x, y}\right)-\mathfrak{u}(A)\right\}, \\
& \left(\mathcal{L}_{+} \mathfrak{u}\right)(A)=2 \sum_{x \in A, y \in A} a(y-x) \mathfrak{u}(A \backslash\{y\}), \\
& \left(\mathcal{L}_{-} \mathfrak{u}\right)(A)=2 \sum_{x \notin A, y \notin A} a(y-x) \mathfrak{u}(A \cup\{y\})
\end{aligned}
$$

and $\mathcal{L}_{s}$ is defined by (3.15). Furthermore, for any function $\mathfrak{u}: \mathcal{E} \rightarrow \mathbb{R}, \mathfrak{T} \mathcal{L}_{\alpha} \mathfrak{u}=\mathfrak{L}_{\alpha} \mathfrak{T} \mathfrak{u}$, provided

$$
\mathfrak{L}_{\alpha}=\mathfrak{L}_{s}+(1-2 \alpha) \mathfrak{L}_{d}+\sqrt{\chi(\alpha)}\left\{\mathfrak{L}_{+}+\mathfrak{L}_{-}\right\}
$$

and, for $A \in \mathcal{E}_{*}, \mathfrak{v}: \mathcal{E}_{*} \rightarrow \mathbb{R}$ a finitely supported function,

$$
\begin{aligned}
& \left(\mathfrak{L}_{d} \mathfrak{v}\right)(A)=\sum_{\substack{x \in A, y \notin A \\
y \neq 0}} a(y-x)\left\{\mathfrak{v}\left(A_{x, y}\right)-\mathfrak{v}(A)\right\}+\sum_{\substack{y \notin A \\
y \neq 0}} a(y)\left\{\mathfrak{v}\left(S_{y} A\right)-\mathfrak{v}(A)\right\}, \\
& \left(\mathfrak{L}_{+} \mathfrak{v}\right)(A)=2 \sum_{\substack{x \in A, y \in A \\
\text { a }}} a(y-x) \mathfrak{v}(A \backslash\{y\})+2 \sum_{x \in A} a(x)\left\{\mathfrak{v}(A \backslash\{x\})-\mathfrak{v}\left(S_{x}[A \backslash\{x\}]\right)\right\}, \\
& \left(\mathfrak{L}_{-} \mathfrak{v}\right)(A)=2 \sum_{\substack{x \notin A, y \notin A \\
x, y \neq 0}} a(y-x) \mathfrak{v}(A \cup\{y\}) .
\end{aligned}
$$


In fact, we know that $\mathfrak{T} \mathcal{L}_{*} \mathfrak{U}=\mathfrak{L}_{*} \mathfrak{T} \mathfrak{u}$, for $*=s, d,+,-$. The following commutative diagram illustrate the relation between the operators recently defined. The first arrow down assigns to each function $u \in \mathcal{C}_{0}$ its Fourier coefficients.

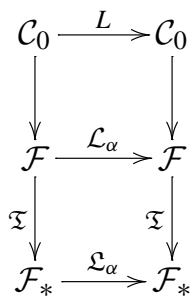

\section{Sector condition, the resolvent equation and some estimates}

We prove in this section three important results. They will be useful to understand the structure of the space $H_{\alpha}$, defined in Section 2. The first one, whose proof is postponed to Appendix B, is related to the so-called sector condition for the generator $L$. The second result, Theorem 4.6, based on an elementary computation, states that all functions in $\mathcal{C}_{0}$ have finite $\|\cdot\|_{-1}$ norm. Finally, the last result states that all local function in $\mathcal{C}_{0}$ may be approximated in $H_{-1}$ by local functions in the range of the generator $L$.

We start with a result related to the sector condition. In this section, $C_{0}$ will be use to denote finite constants depending on the probability $p$.

Theorem 4.1. There exists a finite constant $C_{0}$ such that

$$
\left\langle\mathfrak{L}_{d} \mathfrak{f}, \mathfrak{g}\right\rangle^{2} \leqslant C_{0}\left\langle\mathfrak{f},-\mathfrak{L}_{s} \mathfrak{f}\right\rangle\left\langle\mathfrak{g},-\mathfrak{L}_{s} \mathfrak{g}\right\rangle
$$

for each $\mathfrak{f}, \mathfrak{g}$ in $\mathcal{I}_{*, n}$. The same result remains in force if $\mathfrak{L}_{d}$ is replaced by $\mathfrak{L}_{+}$or $\mathfrak{L}_{-}$with $\mathfrak{g}$ in $\mathcal{I}_{*, n+1}$ and $\mathcal{I}_{*, n-1}$, respectively.

The proof of this result, as well as the proofs of some other estimates concerning the operators $\mathfrak{L}_{+}, \mathfrak{L}_{-}, \mathfrak{L}_{d}$ and $\mathfrak{L}_{s}$ are presented in Appendix B. We state some corollaries that are repeatedly used in this work. The first one is a simple consequence of the definition of $\|\cdot\|_{-1}$ norm for $\mathfrak{f} \in \mathcal{I}_{*, n}$ given in (3.22) and Theorem 4.1.

Corollary 4.2. For $\mathfrak{f} \in \mathcal{I}_{*, n}$ we have that

$$
\left\|\mathfrak{L}_{*} \mathfrak{f}\right\|_{-1} \leqslant C_{0}\|\mathfrak{f}\|_{1},
$$

where $\mathfrak{L}_{*}$ stands for the operators $\mathfrak{L}_{s}, \mathfrak{L}_{d}, \mathfrak{L}_{+}$and $\mathfrak{L}_{-}$.

Corollary 4.3. There exists a finite constant $C_{0}$ such that for every $\mathfrak{f} \in \mathcal{I}_{*, n}$

$$
\left\|\mathfrak{L}_{*} \mathfrak{f}\right\| \leqslant C_{0} n\|\mathfrak{f}\|,
$$

where $\|\cdot\|$ is the usual norm on $L^{2}\left(\mathcal{E}_{*, n}\right)$ and $\mathfrak{L}_{*}$ stands for the operators $\mathfrak{L}_{s}, \mathfrak{L}_{d}, \mathfrak{L}_{+}, \mathfrak{L}_{-}$. Then, for every $\mathfrak{f} \in \mathcal{I}_{*}$, we have

$$
\begin{aligned}
& \left\|\mathfrak{L}_{*} \mathfrak{f}\right\|_{0, k} \leqslant C_{0}\|\mathfrak{f}\|_{0, k+1}, \\
& \left\|\mathfrak{L}_{*} \mathfrak{f}\right\|_{-1, k} \leqslant C_{0}\|\mathfrak{f}\|_{1, k} .
\end{aligned}
$$

Proof. A simple computation shows that $\mathfrak{L}_{s}$ is a bounded operator on each $\mathcal{I}_{*, n}$ and that there exists a constant $C$, depending only on $p$, such that

$$
\left\|\mathfrak{L}_{s} \mathfrak{f}\right\| \leqslant C n\|\mathfrak{f}\|,
$$


for all $\mathfrak{f} \in \mathcal{I}_{*, n}$. The first statement follows from this observation and Theorem 4.1. For the second one, just recall definition of $\|\cdot\|_{0, k},\|\cdot\|_{1, k}$ and $\|\cdot\|_{-1, k}$ done in (3.13), (3.19) and (3.23), respectively. Also observe that $\|\mathfrak{f}\|_{1, k} \leqslant$ $C_{0}\|\mathfrak{f}\|_{0, k+1}$.

Remark 4.4. The previous corollary is saying that $\mathfrak{L}_{\alpha}$ is a bounded operator from $\mathbb{I}_{*}^{k+1}$ to $\mathbb{I}_{*}^{k}\left(\left\|\mathfrak{L}_{*} \mathfrak{f}\right\|_{0, k} \leqslant\right.$ $\left.C_{0}\|\mathfrak{f}\|_{0, k+1}\right)$. From $\left\|\mathfrak{L}_{*} \mathfrak{f}\right\|_{-1, k} \leqslant C_{0}\|\mathfrak{f}\|_{1, k}$ we get that there exists a bounded extension for the operator $\mathfrak{L}_{\alpha}$ from $\mathfrak{H}_{1, k}$ to $\mathfrak{H}_{-1, k}$.

Finally, we get to the last result concerning this kind of estimates. Using the dual representation for the operator $L$, presented in formula (3.28), and performing the same kind of computations presented in the proof of Theorem 4.1, given in Appendix B, we can prove the sector condition. Recall that $\langle\cdot, \cdot\rangle_{\alpha}$ denote the scalar product in $L^{2}\left(v_{\alpha}\right)$, defined in Section 2.

Theorem 4.5. There exists a constant $C$ such that

$$
\langle L f, g\rangle_{\alpha}^{2} \leqslant C\left\langle L^{s} f, f\right\rangle_{\alpha}\left\langle L^{s} g, g\right\rangle_{\alpha},
$$

for every local function $f$ and $g$, for all $\alpha \in[0,1]$.

We turn now to the space $H_{-1}$. We first prove that all function in $\mathcal{C}_{0}$ belong to $H_{-1}$ and then show that they may be approximated by functions in the range of the operator $L$.

Theorem 4.6. If $\mathfrak{u} \in \mathcal{F}_{*, n}$, we have that

$$
\|\mathfrak{u}\|_{-1}<\infty
$$

and, from identity (3.24), we conclude that for $u \in \mathcal{C}_{0}$

$$
\|u\|_{-1}<\infty \text {. }
$$

Proof. We need to prove that there exists a constant $C$ depending on $\mathfrak{u}$ such that

$$
\left(\sum_{A \in \mathcal{E}_{*, n}} \mathfrak{u}(A) \mathfrak{v}(A)\right)^{2} \leqslant C\|\mathfrak{v}\|_{1}^{2}, \quad \text { for every } \mathfrak{v} \in \mathcal{F}_{*, n} .
$$

Denote by $\mathcal{A}$ the support of $\mathfrak{u}: \mathcal{A}=\{A: \mathfrak{u}(A) \neq 0\}$. Since $\mathfrak{u} \in \mathcal{F}_{*, n}, \mathcal{A}$ is a finite set. Considering that $\sum_{B \in \mathcal{A}} \mathfrak{u}(B)=$ 0 and performing a change of variables before apply Schwarz inequality for the last inequality, we get that

$$
\left(\sum_{A \in \mathcal{E}_{*, n}} \mathfrak{u}(A) \mathfrak{v}(A)\right)^{2}=\frac{1}{|\mathcal{A}|^{2}}\left(\sum_{A, B \in \mathcal{A}}(\mathfrak{u}(A)-\mathfrak{u}(B)) \mathfrak{v}(A)\right)^{2} \leqslant C(\mathfrak{u}) \sum_{A, B \in \mathcal{A}}(\mathfrak{v}(A)-\mathfrak{v}(B))^{2} .
$$

Since $A$ and $B$ are fix sets depending on the support of $\mathfrak{u}$, we can go from $A$ to $B$ changing point by point along paths with jumps of positive probability $p$, in order to get

$$
\sum_{A, B \in \mathcal{A}}(\mathfrak{v}(A)-\mathfrak{v}(B))^{2} \leqslant C(\mathfrak{u}, p)\|\mathfrak{v}\|_{1}^{2} .
$$

For the following result, recall the definition of the space $\mathcal{F}_{*}$ given in (3.12).

Theorem 4.7. Given $\mathfrak{G} \in \mathcal{F}_{*}$ there exists a sequence $\mathfrak{h}_{\lambda}$ in $\mathcal{F}_{*}$ such that

$$
\lim _{\lambda \rightarrow 0}\left\|\mathfrak{G}-\mathfrak{L}_{\alpha} \mathfrak{h}_{\lambda}\right\|_{-1,0}=0 .
$$


In view of identity (3.24), the previous result is telling that given a local function $g$ in $\mathcal{C}_{0}$, there exists a sequence $h_{\lambda}$ in $\mathcal{C}_{0}$ such that

$$
\lim _{\lambda \rightarrow 0}\left\|g-L h_{\lambda}\right\|_{-1}=0 .
$$

The proof of Theorem 4.7, presented at the end of this section, is based on the analysis of a resolvent equation associated to the operator $\mathfrak{L}_{\alpha}$.

Proposition 4.8. Given $\mathfrak{g} \in \mathbb{I}_{*}^{k}$ with $\|\mathfrak{g}\|_{-1, k+1}<\infty$ for some $k \geqslant 0$, for each $\lambda>0$ there exists a unique function $\mathfrak{f}_{\lambda} \in \mathcal{I}_{*}$ with $\left\|\mathfrak{f}_{\lambda}\right\|_{0, k+1}<\infty$, that solves the resolvent equation

$$
\lambda \mathfrak{f}_{\lambda}-\mathfrak{L}_{\alpha} \mathfrak{f}_{\lambda}=\mathfrak{g}
$$

in $\mathbb{I}_{*}^{k}$. Furthermore, for each $j \geqslant 0$, there exist constants $C_{j}$, depending on $j$ and the probability $p$, such that

$$
\lambda\left\|\mathfrak{f}_{\lambda}\right\|_{0, j}^{2} \leqslant C_{j}\|\mathfrak{g}\|_{-1, j}^{2} \quad \text { and } \quad\left\|\mathfrak{f}_{\lambda}\right\|_{1, j}^{2} \leqslant C_{j}\|\mathfrak{g}\|_{-1, j}^{2},
$$

with $C_{0}=1$.

The proof of Proposition 4.8 requires some lemmas and some estimates on the operators $\mathfrak{L}_{+}, \mathfrak{L}_{-}, \mathfrak{L}_{d}$ and $\mathfrak{L}_{s}$ presented in Appendix B. Let $\mathcal{J}_{n}$ be the subset of functions in $\mathcal{I}_{*}$ of degree less or equal than $n$ :

$$
\mathcal{J}_{n}=\left\{\mathfrak{f} \in \mathcal{I}_{*}: \mathfrak{f}(A)=0 \text { if }|A|>n\right\} .
$$

Consider the inner product $\langle\cdot, \cdot\rangle_{0,0}$, restricted to this space. Define $\Pi_{n}$ as the projection on $\mathcal{J}_{n}$ :

$$
\Pi_{n}(\mathfrak{f})=\sum_{i \leqslant n} \pi_{i}(\mathfrak{f})
$$

In order to prove Proposition 4.8, we start solving the resolvent equation restricted to $\mathcal{J}_{n}$. Fix $\alpha$ and consider $\mathfrak{L}_{n}=\Pi_{n} \mathfrak{L}_{\alpha} \Pi_{n}$ as an operator from $\mathcal{J}_{n}$ into itself. For $\mathfrak{g}_{n} \in \mathcal{J}_{n}$, consider the resolvent equation given by

$$
\lambda \mathfrak{f}_{\lambda, n}-\mathfrak{L}_{n} \mathfrak{f}_{\lambda, n}=\mathfrak{g}_{n} .
$$

Lemma 4.9. Give $\mathfrak{g}_{n}$ in $\mathcal{J}_{n}$, there exists a unique solution $\mathfrak{f}_{\lambda, n} \in \mathcal{J}_{n}$ for the Eq. (4.6). Furthermore, if $\left\|\mathfrak{g}_{n}\right\|_{-1,0}$ is finite, then

$$
\lambda\left\|\mathfrak{f}_{\lambda, n}\right\|_{0,0}^{2} \leqslant\left\|\mathfrak{g}_{n}\right\|_{-1,0}^{2}, \quad\left\|\mathfrak{f}_{\lambda, n}\right\|_{1,0}^{2} \leqslant\left\|\mathfrak{g}_{n}\right\|_{-1,0}^{2} .
$$

Proof. We first show that the operator $\mathfrak{L}_{n}$ is bounded and non-positive in $\left(\mathcal{J}_{n},\left\langle\langle\cdot, \cdot\rangle_{0,0}\right)\right.$. With these results, existence and uniqueness of solutions of Eq. (4.6) is proved in the usual way. For the first statement, by Corollary 4.3, there exists a finite constant $C(n)$ (also depending on $\alpha$ ) such that for all $\mathfrak{f} \in \mathcal{J}_{n}$

$$
\left\|\mathfrak{L}_{n} \mathfrak{f}\right\|_{0,0} \leqslant C(n)\|\mathfrak{f}\|_{0,0} .
$$

This implies that $\mathfrak{L}_{n}$ is bounded operator in $\mathcal{J}_{n}$. To see that it is non-positive, by Corollary B.2, we have that for all $\mathfrak{f} \in \mathcal{J}_{n},\left\langle\mathfrak{L}_{n} \mathfrak{f}, \mathfrak{f}\right\rangle_{0,0}=\left\langle\left\langle\mathfrak{L}_{s} \mathfrak{f}, \mathfrak{f}\right\rangle_{0,0} \leqslant 0\right.$.

To obtain the bounds, take inner product $\left.(《,,\rangle_{0,0}\right)$ with $\mathfrak{f}_{\lambda, n}$ on both sides of Eq. (4.6) to get that

$$
\lambda\left\|\mathfrak{f}_{\lambda, n}\right\|_{0,0}^{2}+\left\langle-\mathfrak{L}_{n} \mathfrak{f}_{\lambda, n}, \mathfrak{f}_{\lambda, n}\right\rangle_{0,0}=\left\langle\mathfrak{g}, \mathfrak{f}_{\lambda, n}\right\rangle_{0,0} \leqslant\left\|\mathfrak{f}_{\lambda, n}\right\|_{1,0}\|\mathfrak{g}\|_{-1,0} .
$$

Observe that the symmetric part of the operator $\mathfrak{L}_{\alpha}$ is $\mathfrak{L}_{s}$ only when working with $\langle\cdot, \cdot\rangle_{0,0}$.

In fact we can obtain stronger estimates on the solution $\mathfrak{f}_{\lambda, n}$ of the truncated resolvent equation (4.6). The following lemma is taken from Section 5 in [11]. The estimates obtained in Theorem 4.1 are crucial in the proof of this result. 
Lemma 4.10. Let $\mathfrak{f}_{\lambda, n}$ be the solution of the equation

$$
\lambda \mathfrak{f}_{\lambda, n}-\mathfrak{L}_{n} \mathfrak{f}_{\lambda, n}=\mathfrak{g}_{n} .
$$

For any $j \geqslant 1$, there exists a finite constant $C_{j}$, depending on $j$ and the probability $p$, such that

$$
\lambda\left\|\mathfrak{f}_{\lambda, n}\right\|_{0, j}^{2} \leqslant C_{j}\left\|\mathfrak{g}_{n}\right\|_{-1, j}^{2}, \quad\left\|\mathfrak{f}_{\lambda, n}\right\|_{1, j}^{2} \leqslant C_{j}\left\|\mathfrak{g}_{n}\right\|_{-1, j}^{2} .
$$

We are now in the position to prove Proposition 4.8.

Proof of Proposition 4.8. The idea of the proof is to solve the resolvent equation projected into $\mathcal{J}_{n}$ and then to show that the solutions converge to a function $\mathfrak{f}_{\lambda}$ in the domain of the operator $\mathfrak{L}_{\alpha}$ that solves the original equation. Recall the definition of the projection $\Pi_{n}$ and the operator $\mathfrak{L}_{n}$ given in (4.5) and just before (4.6), respectively. By Lemma 4.9, for each $n \geqslant 1$ there exists $\mathfrak{f}_{\lambda, n}$, solution of

$$
\lambda \mathfrak{f}_{\lambda, n}-\mathfrak{L}_{n} \mathfrak{f}_{\lambda, n}=\Pi_{n} \mathfrak{g} .
$$

Since $\left\|\Pi_{n} \mathfrak{g}\right\|_{-1, k} \leqslant\|\mathfrak{g}\|_{-1, k}$, by Lemma 4.10

$$
\begin{aligned}
& \left\|\mathfrak{f}_{\lambda, n}\right\|_{1, k}^{2} \leqslant C_{k}\left\|\Pi_{n} \mathfrak{g}\right\|_{-1, k}^{2} \leqslant C_{k}\|\mathfrak{g}\|_{-1, k}^{2}, \\
& \lambda\left\|\mathfrak{f}_{\lambda, n}\right\|_{0, k}^{2} \leqslant C_{k}\left\|\Pi_{n} \mathfrak{g}\right\|_{-1, k}^{2} \leqslant C_{k}\|\mathfrak{g}\|_{-1, k}^{2} .
\end{aligned}
$$

In particular, for each $\lambda, \mathfrak{f}_{\lambda, n}$ is a bounded sequence for \|\|$_{0, k+1}$. Then, there exists a subsequence $\mathfrak{f}_{\lambda, n_{j}}$ converging weakly to some function $\mathfrak{f}_{\lambda}$ with $\left\|\mathfrak{f}_{\lambda}\right\|_{0, k+1}$ finite. We claim that the limit is a solution of the resolvent equation (4.2). From Remark 4.4, we have that $\mathfrak{L}_{\alpha}: \mathbb{I}_{*}^{k+1} \rightarrow \mathbb{I}_{*}^{k}$ is a bounded operator and so preserves weakly convergent sequences. This means that $\mathfrak{L}_{\alpha} \mathfrak{f}_{\lambda, n_{j}}$ converges weakly to $\mathfrak{L}_{\alpha} \mathfrak{f}_{\lambda}$ in $\mathbb{I}_{*}^{k}$. We also have that $\mathfrak{f}_{\lambda, n_{j}}$ converges weakly to $\mathfrak{f}_{\lambda}$ in $\mathbb{I}_{*}^{k}$ and that $\Pi_{n} \mathfrak{g}$ converges to $\mathfrak{g}$. All the previous convergences imply that $\mathfrak{f}_{\lambda}$ is solution of the resolvent equation on $\mathbb{I}_{*}^{k}$ :

$$
\lambda \mathfrak{f}_{\lambda}-\mathfrak{L}_{\alpha} \mathfrak{f}_{\lambda}=\mathfrak{g} .
$$

Take inner product $\langle\cdot \cdot \cdot \cdot\rangle_{0,0}$ with $\mathfrak{f}_{\lambda}$ in the previous expression and considering that $\mathfrak{f}$ belongs to $\mathbb{I}_{*}^{1}$, use Remark B.3 to get that

$$
\left\|\mathfrak{f}_{\lambda}\right\|_{1,0}^{2} \leqslant\|\mathfrak{g}\|_{-1,0}^{2}, \quad \lambda\left\|\mathfrak{f}_{\lambda}\right\|_{0,0}^{2} \leqslant\|\mathfrak{g}\|_{-1,0}^{2} .
$$

Uniqueness of solution follows from the fact that $\lambda-\mathfrak{L}_{\alpha}$ is a strictly positive operator on $\mathbb{I}_{*}^{k}$, for $k \geqslant 1$. To conclude the proof of Proposition 4.8, it remains to get the bounds announced. Once we have solution for the resolvent equation, the prove of Lemma 4.10 works. Then we get that, for $j \geqslant 1$,

$$
\begin{aligned}
& \lambda\left\|\mathfrak{f}_{\lambda}\right\|_{0, j}^{2} \leqslant C_{j}\|\mathfrak{g}\|_{-1, j}^{2}, \\
& \left\|\mathfrak{f}_{\lambda}\right\|_{1, j}^{2} \leqslant C_{j}\|\mathfrak{g}\|_{-1, j}^{2},
\end{aligned}
$$

where $C_{j}$ are the constants appearing in Lemma 4.10.

We conclude this section with the proof of Theorem 4.7.

Proof of Theorem 4.7. Given $\mathfrak{G} \in \mathcal{F}_{*}$, we know by Theorem 4.6 that $\|\mathfrak{G}\|_{-1, k}<\infty$ for all $k$. Then, by Proposition 4.8, there exists $\mathfrak{f}_{\lambda}$ solution of the resolvent equation

$$
\lambda \mathfrak{f}_{\lambda}-\mathfrak{L}_{\alpha} \mathfrak{f}_{\lambda}=-\mathfrak{G},
$$

in $\mathbb{I}_{*}^{k}$, for all $k \geqslant 0$. We will see that

$$
\lim _{\lambda \rightarrow 0}\left\|\mathfrak{G}-\mathfrak{L}_{\alpha} \mathfrak{f}_{\lambda}\right\|_{-1,0}=0 .
$$


Then approximate $\mathfrak{L}_{\alpha} \mathfrak{f}_{\lambda}$ by $\mathfrak{L}_{\alpha} \mathfrak{h}_{\lambda}$ in $\|\cdot\|_{-1,0}$ with $\mathfrak{h}_{\lambda}$ in $\mathcal{F}_{*}$ close to $\mathfrak{f}_{\lambda}$ in the $\|\cdot\|_{1,0}$ norm.

To prove convergence (4.12), we start showing that $\mathfrak{L}_{\alpha} \mathfrak{f}_{\lambda}$ is bounded for the $\|\cdot\|_{-1,0}$ norm. Then we characterize weak limits. Finally we prove that $\mathfrak{L}_{\alpha} \mathfrak{f}_{\lambda}$ is Cauchy for the $\|\cdot\|_{-1,0}$ norm.

Take inner product $\left(\left\langle\langle\cdot, \cdot\rangle_{0,0}\right)\right.$ with $\mathfrak{f}_{\lambda}$ on both sides of Eq. (4.11) and recall that $\left\langle-\mathfrak{G}, \mathfrak{f}_{\lambda}\right\rangle_{0,0} \leqslant\|\mathfrak{G}\|_{-1,0}\left\|f_{\lambda}\right\|_{1,0}$, to get that

$$
\begin{aligned}
& \left\|\mathfrak{f}_{\lambda}\right\|_{1,0} \leqslant\|\mathfrak{G}\|_{-1,0}, \\
& \lambda\left\langle\mathfrak{f}_{\lambda}, \mathfrak{f}_{\lambda}\right\rangle_{0,0} \leqslant\|\mathfrak{G}\|_{-1,0}^{2} .
\end{aligned}
$$

Observe, in particular, that $\lambda \mathfrak{f}_{\lambda}$ converges to 0 in the $\|\cdot\|_{0,0}$ norm. As $\mathfrak{L}_{\alpha}$ is a linear combination of $\mathfrak{L}_{*}$, for $*=s, d,+,-$, use Corollary 4.3 and Proposition 4.8, to get that

$$
\left\|\mathfrak{L}_{\alpha} \mathfrak{f}_{\lambda}\right\|_{-1,0} \leqslant C(\alpha)\left\|\mathfrak{f}_{\lambda}\right\|_{1,0} \leqslant C(\alpha)\|\mathfrak{G}\|_{-1,0} .
$$

Therefore $\mathfrak{L}_{\alpha} \mathfrak{f}_{\lambda}$ is bounded for the $\|\cdot\|_{-1,0}$ norm. As in Lemma 2.8 of [6], we can prove that

(1) If $\mathfrak{L}_{\alpha} \mathfrak{f}_{\lambda_{j}}$ converges weakly in $\|\cdot\|_{-1,0}$ norm as $\lambda_{j} \downarrow 0$, then the limit is $\mathfrak{G}$.

(2) There exists $\mathfrak{f} \in \mathfrak{H}_{1,0}$ such that $\mathfrak{f}_{\lambda}$ converges strongly to $\mathfrak{f}$ in $\mathfrak{H}_{1,0}$.

Since, by Corollary 4.3,

$$
\left\|\mathfrak{L}_{\alpha} \mathfrak{f}_{\lambda}-\mathfrak{L}_{\alpha} \mathfrak{f}_{\tilde{\lambda}}\right\|_{-1,0} \leqslant C(\alpha)\left\|\mathfrak{f}_{\lambda}-\mathfrak{f}_{\tilde{\lambda}}\right\|_{1,0},
$$

and since $\mathfrak{f}_{\lambda}$ converges strongly in $\mathfrak{H}_{1,0}, \mathfrak{L}_{\alpha} \mathfrak{f}_{\lambda}$ is Cauchy for $\|\cdot\|_{-1,0}$. Considering that we have just characterized all weak limit points, it follows that $\mathfrak{L}_{\alpha} \mathfrak{f}_{\lambda}$ converges strongly to $\mathfrak{G}$ in $\|\cdot\|_{-1,0}$ :

$$
\left\|\mathfrak{L}_{\alpha} \mathfrak{f}_{\lambda}-\mathfrak{G}\right\|_{-1,0} \rightarrow 0 .
$$

Take $\mathfrak{h}_{\lambda}$ in $\mathfrak{F}_{*}$ such that $\lim _{\lambda \rightarrow 0}\left\|\mathfrak{f}_{\lambda}-\mathfrak{h}_{\lambda}\right\|_{1,0}=0$. From Corollary 4.3 , we get that $\left\|\mathfrak{L}_{\alpha} \mathfrak{f}_{\lambda}-\mathfrak{L}_{\alpha} \mathfrak{h}_{\lambda}\right\|_{-1,0} \leqslant$ $C(\alpha)\left\|\mathfrak{f}_{\lambda}-\mathfrak{h}_{\lambda}\right\|_{1,0}$. Since $\mathfrak{L}_{\alpha} \mathfrak{f}_{\lambda}$ converges to $\mathfrak{G}$ in $\mathfrak{H}_{-1,0}$ we can conclude that $\mathfrak{L}_{\alpha} \mathfrak{h}_{\lambda}$ also converges to $\mathfrak{G}$ in $\mathfrak{H}_{-10}$ :

$$
\lim _{\lambda \rightarrow 0}\left\|\mathfrak{L}_{\alpha} \mathfrak{h}_{\lambda}-\mathfrak{G}\right\|_{-1,0}=0 .
$$

Remark 4.11. Recall that in Remark 4.4 we said that the operator $\mathfrak{L}_{\alpha}$ admits an extension from $\mathfrak{H}_{1,0}$ to $\mathfrak{H}_{-1,0}$. Some how, we are saying that we can solve the equation $\mathfrak{L}_{\alpha} \mathfrak{f}=\mathfrak{G}$ with $\mathfrak{f}$ in $\mathfrak{H}_{1,0}$.

\section{The space $H_{\alpha}$}

We prove in this section a structure theorem for the Hilbert space of variances, $H_{\alpha}$, that allows to derive, in the next section, an explicit formula for the diffusion coefficient $D_{i, j}(\alpha)$. Recall that $\sigma=\left(\sigma_{i, j}\right)_{1 \leqslant i, j \leqslant d}$, is the matrix defined by $\sigma_{i, j}=\sum_{y} s(y) y_{i} y_{j}$ and that $\chi(\alpha)=\alpha(1-\alpha)$. For $\alpha \in(0,1)$ and $h \in \mathcal{C}_{0}$ consider

$$
\langle h\rangle_{\alpha}=\sup _{a \in \mathbb{R}^{d}}\left\{2 \sum a_{i}\langle h\rangle_{\alpha, i}-\frac{1}{2} \chi(\alpha) a^{*} \sigma a\right\}+\sup _{g \in \mathcal{C}_{0}}\left\{2\left\langle\langle g, h\rangle_{\alpha, 0}-\left\langle-L^{s} g, g\right\rangle_{\alpha, 0}\right\},\right.
$$

where

$$
\langle h\rangle_{\alpha, i}=\sum_{x \in \mathbb{Z}^{d}}\left\langle x_{i} \eta(x) h\right\rangle_{\alpha}, \quad\left\langle\langle h, g\rangle_{\alpha, 0}=\sum_{x \in \mathbb{Z}^{d}}\left\langle\tau_{x} h, g\right\rangle_{\alpha}\right.
$$

and $a^{*} \sigma a$ is matrix product with $a^{*}$ for a line vector in $\mathbb{R}^{d}$. Observe that in each of the previous summations, we only need to consider a finite number of terms since $h \in \mathcal{C}_{0}$. 
It will be useful to denote by $\|h\|_{\alpha}$ the first term on the right hand side of (5.1).

We prove in Lemma 5.2 that $\langle h\rangle_{\alpha}<\infty$ for every $h$ in $\mathcal{C}_{0}$. Recall that $H_{\alpha}$ is the Hilbert space induced by the semi-norm $\langle\cdot\rangle_{\alpha}^{1 / 2}$ on $\mathcal{C}_{0}$. In this section we show that every element in $H_{\alpha}$ can be approximated by $\sum D_{j}\left[\eta\left(e_{j}\right)-\right.$ $\eta(0)]+L u$ for $D$ in $\mathbb{R}^{d}$ and $u \in \mathcal{C}_{0}$. The main result is the following.

\section{Theorem 5.1.}

$$
H_{\alpha}=\overline{\left\{\eta\left(e_{j}\right)-\eta(0), 1 \leqslant j \leqslant d\right\} \oplus L \mathcal{C}_{0}} .
$$

In fact, given $g \in \mathcal{C}_{0}$, there exist unique $\left\{D_{j}(\alpha), 1 \leqslant j \leqslant d\right\}$ such that

$$
\left.\inf _{u \in \mathcal{C}_{0}} \|\left\langle g+\sum_{j=1}^{d} D_{j}(\alpha)\left[\eta\left(e_{j}\right)-\eta(0)\right]-L u\right\rangle\right\rangle_{\alpha}=0 .
$$

Furthermore, consider $\mathfrak{m}_{j} \in \mathcal{F}_{*, 1}$ given by

$$
\mathfrak{m}_{j}(\{x\})=2 a(x) x_{j} .
$$

Then

$$
D_{j}(\alpha)=\frac{-1}{\chi(\alpha)}\left\langle\left\langle\pi_{1} g\right\rangle_{\alpha, j}+\lim _{\lambda \rightarrow 0}\left\langle\left\langle\mathfrak{f}_{\lambda}, \mathfrak{m}_{j}\right\rangle_{0,0},\right.\right.
$$

where $\mathfrak{f}_{\lambda}$ solves the resolvent equation

$$
\lambda \mathfrak{f}_{\lambda}-\mathfrak{L}_{\alpha} \mathfrak{f}_{\lambda}=-\mathfrak{T} \mathfrak{g}
$$

in $\mathcal{I}_{*}, \mathfrak{g}$ denotes the Fourier coefficients of $g$ and $\mathfrak{T}$ is the operator defined in (3.5).

The proof of this result is presented at the end of this section.

We start proving that $\langle h\rangle_{\alpha}$ is finite for $h \in \mathcal{C}_{0}$.

Lemma 5.2. If $h \in \mathcal{C}_{0}$ then $\langle h\rangle_{\alpha}<\infty$.

Proof. We need to see that each term appearing in definition (5.1) is finite if $h \in \mathcal{C}_{0}$. For the first one, recall that

$$
\|h\|_{\alpha}=\sup _{a \in \mathbb{R}^{d}}\left\{2 \sum a_{i}\langle h\rangle_{\alpha, i}-\frac{1}{2} \alpha\{1-\alpha\} a^{*} \sigma a\right\} .
$$

This term may be computed since the matrix $\sigma$ has an inverse. Put $\mathbb{H}$ for the column vector in $\mathbb{R}^{d}$ whose coordinates are given by $\mathbb{H}_{i}=\langle h\rangle_{\alpha, i}$. Denote by $\mathbb{H}^{*}$ the transposition of $\mathbb{H}$ and by $\sigma^{-1}$ the inverse of the matrix $\sigma$. Then we have that

$$
\|h\|_{\alpha}=2 / \chi(\alpha) \mathbb{H}^{*} \sigma^{-1} \mathbb{H} \text {. }
$$

The second term appearing in definition (5.1) is $\|h\|_{-1}^{2}$, defined in (3.21). In Theorem 4.6 we claimed that $\|h\|_{-1}<$ $\infty$ if $h \in \mathcal{C}_{0}$. This completes the proof of the present lemma.

Observe that the semi-norm $\langle\cdot\rangle_{\alpha}$ depends only on the symmetric part of the generator. It may be proved, as in Chapter 7 of [2], that given a cylinder function $h$ in $\mathcal{C}_{0}$ and a sequence of positive integers $K_{\ell}$ such that $0 \leqslant K_{\ell} \leqslant(2 \ell+1)^{d}$ and $\lim _{\ell \rightarrow \infty} K_{\ell} /(2 \ell)^{d}=\alpha$, then

$$
\lim _{\ell \rightarrow \infty}(2 \ell)^{-d}\left\langle\left(-L_{\Lambda_{\ell}}^{s}\right)^{-1} \sum_{|x| \leqslant \ell_{h}} \tau_{x} h, \sum_{|x| \leqslant \ell_{h}} \tau_{x} h\right\rangle_{\ell, K_{\ell}}=\langle h\rangle_{\alpha},
$$


where $L_{\Lambda_{\ell}}^{s}$ represents the symmetric part of the generator restricted to the box $\Lambda_{\ell}=[-\ell, \ell]^{d} \cap \mathbb{Z}^{d}, \ell_{h}$ is such that $\sum_{|x| \leqslant \ell_{h}} \tau_{x} h$ is measurable with respect to $\left\{\eta(x) ; x \in \Lambda_{\ell}\right\}, \ell_{h} / \ell \rightarrow 1$ and we are considering the uniform measure on the space of configurations in the box $\Lambda_{\ell}$ with $K_{\ell}$ particles. Observe that, since $h \in \mathcal{C}_{0},\langle h\rangle_{\ell, K_{\ell}}=0$ for all $K_{\ell}$, for $\ell$ big enough. This fact guarantees that the left hand side inside the brackets in (5.5) is well defined.

Recall from (2.3) that $W_{x, x+y}$ and $W_{x, x+y}^{*}$ stand for the current of the process and the dual process, respectively. For $1 \leqslant i \leqslant d$, let

$$
\begin{aligned}
& \nabla_{i}(\eta)=\sum_{y} y_{i}[\eta(0)-\eta(y)] s(y), \\
& W_{i}^{*}(\eta)=\frac{1}{2} \sum_{y} y_{i} W_{0, y}^{*}, \quad W_{i}(\eta)=\frac{1}{2} \sum_{y} y_{i} W_{0, y} .
\end{aligned}
$$

As in [5], we present some identities that can be formally derived from (5.5), and the relations $L\left[\sum x_{i} \eta(x)\right]=$ $\sum \tau_{x} W_{i}$ and $L^{s}\left[\sum x_{i} \eta(x)\right]=1 / 2 \sum \tau_{x} \nabla_{i}$. They will help the reader to follow the references appearing in this section.

$$
\begin{array}{ll}
\left\langle\left\langle L^{*} g, \nabla_{i}\right\rangle_{\alpha}=-2\left\langle\left\langle W_{i}, g\right\rangle_{\alpha, 0},\right.\right. & \left\langle\left\langle L g, \nabla_{i}\right\rangle_{\alpha}=-2\left\langle\left\langle W_{i}^{*}, g\right\rangle_{\alpha, 0},\right.\right. \\
\left\langle\nabla_{i}, h\right\rangle_{\alpha}=-2\langle h\rangle_{\alpha, i}, & \left\langle\left\langle\nabla_{i}, L^{s} g\right\rangle_{\alpha}=0,\right. \\
\left\langle\nabla_{i}, \nabla_{k}\right\rangle_{\alpha}=2 \chi(\alpha) \sigma_{i, k}, & \left\langle\left\langle L^{s} f, h\right\rangle_{\alpha}=-\langle f f, h\rangle_{\alpha, 0} .\right.
\end{array}
$$

A crucial difference between symmetric nongradient systems (as the symmetric generalized exclusion process) and asymmetric ones appears when we want to compute $\langle L h, L h\rangle_{\alpha}$. In the symmetric case, the last line in (5.8) gives us an explicit formula for this object. In the asymmetric case, the sector condition proved in Theorem 4.5 allows to control $\langle L h, L h\rangle_{\alpha}$ in terms of $\left\langle\left\langle L^{s} h, L^{s} h\right\rangle_{\alpha}\right.$. The following proposition is a consequence of Theorem 4.5 and (5.5).

Proposition 5.3. There exists a constant $C$ depending only on the probability $p$ such that

$$
\left\langle L^{*} h, L^{*} h\right\rangle_{\alpha} \leqslant C\left\langle\left\langle L^{s} h, L^{s} h\right\rangle_{\alpha}\right.
$$

and

$$
\langle L h, L h\rangle_{\alpha} \leqslant C\left\langle\left\langle L^{s} h, L^{s} h\right\rangle_{\alpha},\right.
$$

for any function $h \in \mathcal{C}_{0}$.

We start studying the spaces involved in decomposition (5.2).

Lemma 5.4. $\left\{\left[\eta\left(e_{i}\right)-\eta(0)\right], 1 \leqslant i \leqslant d\right\}$ are linearly independent in $H_{\alpha}$.

Proof. From $\left\langle\left\langle\nabla_{i}, \nabla_{k}\right\rangle_{\alpha}=2 \chi(\alpha) \sigma_{i, k}\right.$ we get that $\left\{\nabla_{i}, 1 \leqslant i \leqslant d\right\}$ are linearly independent in $H_{\alpha}$. On the other hand, since $h=\tau_{x} h$ in $H_{\alpha}$ for any $h \in \mathcal{C}_{0}$ and $x \in \mathbb{Z}^{d}$, we get that

$$
\nabla_{i}=\sum_{y} y_{i}[\eta(0)-\eta(y)] s(y)=\sum_{y, j} y_{i} y_{j}\left[\eta(0)-\eta\left(e_{j}\right)\right] s(y)=\sum_{j} \sigma_{i, j}\left[\eta(0)-\eta\left(e_{j}\right)\right] .
$$

This means that $\left\{\nabla_{i}, 1 \leqslant i \leqslant d\right\}$ and $\left\{\left[\eta\left(e_{i}\right)-\eta(0)\right], 1 \leqslant i \leqslant d\right\}$ generate the same linear space in $H_{\alpha}$.

The following result is, essentially, Lemma 3.6 in [3]. It states that the spaces generating $H_{\alpha}$ in (5.2) are in direct sum. 
Lemma 5.5. The linear space generated by $\left\{\nabla_{i}, 1 \leqslant i \leqslant d\right\}$ in $H_{\alpha}$ does not intersect the closure of $L \mathcal{C}_{0}$ :

$$
\left\{\sum_{i=1}^{d} a_{i} \nabla_{i}: a \in \mathbb{R}^{d}\right\} \cap \overline{L \mathcal{C}_{0}}=\{0\} .
$$

To complete this section, it remains to prove that the spaces presented in the decomposition (5.2) generate the space $H_{\alpha}$. Recall that in the space of Fourier coefficients, we have defined the projections operators $\pi_{n}: L^{2}(\mathcal{E}) \rightarrow$ $L^{2}\left(\mathcal{E}_{n}\right)$ in (3.10). We also use $\pi_{n}$ to denote the projection operator from $L^{2}\left(v_{\alpha}\right)$ to the subspace $\mathcal{G}_{n}$, defined in Section 3. If $f=\sum_{A \in \mathcal{E}} \mathfrak{f}(A) \Psi_{A}$, we use $\pi_{n} f$ for $\sum_{A \in \mathcal{E}_{n}} \mathfrak{f}(A) \Psi_{A}$. With this notation, we have that $f=\sum \pi_{n} f$. Observe that $\pi_{0} f=E_{v_{\alpha}}[f]=0$ since we work with functions $f \in \mathcal{C}_{0}$. We say that a cylinder function has degree $n$ if all its Fourier coefficients are zero, except those of degree $n: f=\sum_{A \in \mathcal{E}_{n}} \mathfrak{f}(A) \Psi_{A}$.

The following lemma shows that $\|h\|_{\alpha}$ is relates to $\pi_{1} h$, the degree one part of the function, while the second term in the right hand side of (5.1) is related to $h-\pi_{1} h$.

Lemma 5.6. For every $h \in \mathcal{C}_{0}$, we have that

$$
\left\langle\langle h\rangle_{\alpha}=\left\|\pi_{1} h\right\|_{\alpha}+\left\langle\left\langle\mathfrak{T}\left(\mathfrak{h}-\pi_{1} \mathfrak{h}\right\rangle\right\rangle_{-1,0},\right.\right.
$$

where $\mathfrak{h}$ are the Fourier coefficients of $h$ and $\mathfrak{T}$ is the operator defined in (3.5).

Proof. Functions of different degrees are orthogonal in $H_{\alpha}$ since the operator $L^{s}$ preserves degree. Then,

$$
\langle h\rangle_{\alpha}=\left\langle\left\langle\pi_{1} h\right\rangle_{\alpha}+\left\langle\left\langle h-\pi_{1} h\right\rangle_{\alpha} .\right.\right.
$$

We claim that $\left\langle\left\langle\pi_{1} h\right\rangle_{\alpha}=\left\|\pi_{1} h\right\|_{\alpha}\right.$ and $\left\langle h-\pi_{1} h\right\rangle_{\alpha}=\left\langle\langle\mathfrak{T h}\rangle_{-1,0}\right.$. For the first identity, observe that if $\mathfrak{h}$ and $\mathfrak{g}$ denote the Fourier coefficients of $h$ and $g$, respectively, we have that

$$
\left\langle\left\langle g, \pi_{1} h\right\rangle_{\alpha, 0}=\sum_{x \in \mathbb{Z}^{d}} \mathfrak{h}(x, \alpha) \sum_{y \in \mathbb{Z}^{d}} \mathfrak{g}(y, \alpha)=0,\right.
$$

by (3.2). Then, the second term in (5.1) is equal to zero if $h$ has degree one. So that $\left\langle\left\langle\pi_{1} h\right\rangle_{\alpha}=\left\|\pi_{1} h\right\|_{\alpha}\right.$.

For the second identity, observe that for $\Psi_{A}$ with $|A| \geqslant 2$, we have

$$
\left\langle\Psi_{A}\right\rangle_{\alpha, i}=\sum_{x \in \mathbb{Z}^{d}}\left\langle x_{i} \eta(x) \Psi_{A}\right\rangle_{\alpha}=0
$$

Since $h-\pi_{1} h=\sum_{n \geqslant 2} \sum_{A \in \mathcal{E}_{n}} \mathfrak{h}(A) \Psi_{A}$, we get that $\left\langle h-\pi_{1} h\right\rangle_{\alpha, i}=0$. In particular, $\left\langle h-\pi_{1} h\right\rangle_{\alpha}=\| \mathfrak{T}(\mathfrak{h}-$ $\left.\pi_{1} \mathfrak{h}\right)\left\|_{-1,0}=\right\| \mathfrak{T h} \|_{-1,0}$ since $\mathfrak{T} \pi_{1} \mathfrak{h}=0$, as we observed at the first point of Remark 3.1.

Proof of Theorem 5.1. We need to prove that given $g \in \mathcal{C}_{0}$, there exist unique $\left\{D_{j}(\alpha), 1 \leqslant j \leqslant d\right\}$ such that

$$
\left.\inf _{u \in \mathcal{C}_{0}} \|\left\langle g+\sum_{j=1}^{d} D_{j}(\alpha)\left[\eta\left(e_{j}\right)-\eta(0)\right]-L u\right\rangle\right\rangle_{\alpha}=0 .
$$

Uniqueness for $D_{j}$ follows from Lemmas 5.4 and 5.5. From Lemma 5.6, we know that

$$
\begin{aligned}
& \left\langle\left\langle g+\sum_{j=1}^{d} D_{j}(\alpha)\left[\eta\left(e_{j}\right)-\eta(0)\right]-L u\right\rangle \|_{\alpha}\right. \\
& \quad=\left\|\pi_{1} g+\sum_{j=1}^{d} D_{j}(\alpha)\left[\eta\left(e_{j}\right)-\eta(0)\right]-\pi_{1} L u\right\|_{\alpha}+\left\|\mathfrak{T} \mathfrak{g}-\mathfrak{L}_{\alpha} \mathfrak{T} \mathfrak{u}\right\|_{-1,0},
\end{aligned}
$$


where, as usual, $\mathfrak{u}$ and $\mathfrak{g}$ are the Fourier coefficients of $u$ and $g$, respectively. At this point we realize that is convenient to work in the space $\mathcal{I}_{*}$. Put $\mathfrak{G}=\mathfrak{T} \mathfrak{g}$ and $\mathfrak{f}=\mathfrak{T} \mathfrak{u}$. From Theorem 4.7, we know that there exists $\mathfrak{h}_{\lambda}$ in $\mathcal{F}_{*}$ such that $\left\|\mathfrak{G}-\mathfrak{L}_{\alpha} \mathfrak{h}_{\lambda}\right\|_{-1,0}$ goes to zero as $\lambda$ goes to zero.

Take $u_{\lambda} \in \mathcal{C}_{0}$ such that $\mathfrak{T}\left(\mathfrak{u}_{\lambda}\right)=\mathfrak{h}_{\lambda}$, as in (3.1). We have that

$$
\lim _{\lambda \rightarrow 0}\left\|\left\langle g+\sum D_{j}(\alpha)\left[\eta\left(e_{j}\right)-\eta(0)\right]-L u_{\lambda}\right\rangle\right\|_{\alpha}=\lim _{\lambda \rightarrow 0}\|\|_{1} g+\sum D_{j}(\alpha)\left[\eta\left(e_{j}\right)-\eta(0)\right]-\pi_{1} L u_{\lambda}\|\|_{\alpha},
$$

so we need to find $D_{j}(\alpha)$ such that

$$
\lim _{\lambda \rightarrow 0}\left\|\pi_{1} g+\sum D_{j}(\alpha)\left[\eta\left(e_{j}\right)-\eta(0)\right]-\pi_{1} L u_{\lambda}\right\| \|_{\alpha}=0 .
$$

Looking at the explicit formula for $\|\cdot\|_{\alpha}$ given in the proof of Lemma 5.2, we reduce the problem to find $D_{j}(\alpha)$ such that $\left\langle\pi_{1} g+\sum D_{j}\left[\eta\left(e_{j}\right)-\eta(0)\right]-\pi_{1} L u_{\lambda}\right\rangle_{\alpha, k}$ goes to zero for $k=1, \ldots, d$, as $\lambda$ goes to 0 . Since $\left\langle\eta \eta\left(e_{j}\right)-\eta(0)\right\rangle_{\alpha, k}=\delta_{k, j} \chi(\alpha)$, we need to prove that

$$
D_{j}(\alpha)=\frac{1}{\chi(\alpha)}\left[-\left\langle\left\langle\pi_{1} g\right\rangle_{\alpha, j}+\lim _{\lambda \rightarrow 0}\left\langle\left\langle\pi_{1} L u_{\lambda}\right\rangle_{\alpha, j}\right]\right.\right.
$$

is well defined. From the dual representation for the operator $L$ obtained in Section 3, we have that

$$
\begin{aligned}
\pi_{1}(L u)= & \sum_{x \in \mathbb{Z}^{d}} \mathcal{L}_{s} \mathfrak{u}_{1}(x) \Psi_{x}+(1-2 \alpha) \sum_{x \in \mathbb{Z}^{d}} \mathcal{L}_{d} \mathfrak{u}_{1}(x) \Psi_{x} \\
& +\sqrt{\chi(\alpha)} \sum_{x \in \mathbb{Z}^{d}} \mathcal{L}_{-} \mathfrak{u}_{2}(x) \Psi_{x}+\sqrt{\chi(\alpha)} \sum_{x \in \mathbb{Z}^{d}} \mathcal{L}_{+} \mathfrak{u}_{0}(x) \Psi_{x}
\end{aligned}
$$

where the operators involved in the previous expression where defined in (3.29).

By construction, $\mathfrak{u}_{\lambda}(x)=0$ for every $x$ in $\mathbb{Z}^{d}$ (see Remark 3.1) and also

$$
\mathfrak{u}_{\lambda}(x, y)= \begin{cases}1 / 2 \mathfrak{h}_{\lambda}(x) & \text { if } y=0, \quad x \neq 0, \\ 1 / 2 \mathfrak{h}_{\lambda}(y) & \text { if } x=0, \quad y \neq 0\end{cases}
$$

Then, an elementary computation gives that

$$
\pi_{1} L u_{\lambda}=\sqrt{\chi(\alpha)} \sum_{x \in \mathbb{Z}^{d}} \mathcal{L}_{-} \mathfrak{u}_{\lambda, 2}(x) \Psi_{x}=\sum a(x) \mathfrak{h}_{\lambda}(x)\{\eta(x)-\eta(0)\} .
$$

Since

$$
\langle\eta(x)-\eta(0)\rangle_{\alpha, j}=x_{j} \chi(\alpha)
$$

we have that

$$
\left\langle\pi_{1} L u_{\lambda}\right\rangle_{\alpha, j}=\chi(\alpha) \sum_{x \in \mathbb{Z}^{d}} a(x) \mathfrak{h}_{\lambda}(x) x_{j},
$$

so that

$$
\frac{1}{\chi(\alpha)} \lim _{\lambda \rightarrow 0}\left\langle\left\langle\pi_{1} L u_{\lambda}\right\rangle_{\alpha, j}=\lim _{\lambda \rightarrow 0}\left\langle\left\langle\mathfrak{h}_{\lambda}, \mathfrak{m}_{j}\right\rangle_{0,0},\right.\right.
$$

with $\mathfrak{m}_{j}$ defined in (5.3). Recall, from Theorem 4.7, that we took $\mathfrak{h}_{\lambda} \in \mathcal{F}_{*}$ such that $\left\|\mathfrak{h}_{\lambda}-\mathfrak{f}_{\lambda}\right\|_{1,0}$ goes to zero as $\lambda$ goes to zero, with $\mathfrak{f}_{\lambda}$ solution of the resolvent equation (4.11). Since $\mathfrak{m}_{j}$ belongs to $\mathfrak{H}_{-1,0}$, we have that

$$
\lim _{\lambda \rightarrow 0}\left\langle\mathfrak{h}_{\lambda}, \mathfrak{m}_{j}\right\rangle_{0,0}=\lim _{\lambda \rightarrow 0}\left\langle\left\langle\mathfrak{f}_{\lambda}, \mathfrak{m}_{j}\right\rangle_{0,0} .\right.
$$


This last limit exists since $\mathfrak{f}_{\lambda}$ is converging in $\|\cdot\|_{1,0}$ (from the proof of Theorem 4.7) and $\mathfrak{m}_{j}$ belongs to $\mathfrak{H}_{-1,0}$. Finally,

$$
D_{j}(\alpha)=\frac{-1}{\chi(\alpha)}\left\langle\left\langle\pi_{1} g\right\rangle_{\alpha, j}+\lim _{\lambda \rightarrow 0}\left\langle\left\langle\mathfrak{f}_{\lambda}, \mathfrak{m}_{j}\right\rangle_{0,0},\right.\right.
$$

with $\mathfrak{f}_{\lambda}$ solution of (4.11), is well defined and solves the problem.

\section{Regularity of the diffusion coefficient}

The goal of this section is to prove Theorem 2.1. In order to do that, we start deriving a convenient expression for the diffusion coefficient. This new formulation, together with an appropriate way of differentiating, allow to prove the regularity properties of the diffusion coefficient.

As we mentioned in Section 2, the techniques developed to prove hydrodynamic behavior of nongradient systems show that the diffusion coefficient $D_{i, j}(\alpha)$ of the hydrodynamic equation for the mean zero simple exclusion process is characterized by

$$
\inf _{u \in \mathcal{C}_{0}} \|\left\langle W_{i}+\sum_{j} D_{i, j}(\alpha)\left[\eta\left(e_{j}\right)-\eta(0)\right]-L u \|_{\alpha}=0,\right.
$$

where $W_{i}$ and its Fourier coefficients, $\mathfrak{W}_{i}(A)=\mathfrak{W}_{i}(A, \alpha)$, are given by

$$
W_{i}(\eta)=1 / 2 \sum_{y} y_{i} W_{0, y}=1 / 2 \sum_{y} y_{i} \eta(-y) p(y)-\sum_{y} \eta(0) \eta(y) a(y) y_{i}
$$

and

$$
\mathfrak{W}_{i}(A, \alpha)= \begin{cases}-\sqrt{\chi(\alpha)} y_{i}(1 / 2 p(-y)+\alpha a(y)) & \text { if } A=\{y\}, \\ -\chi(\alpha) a(y) y_{i} & \text { if } A=\{0, y\}, \\ 0 & \text { otherwise. }\end{cases}
$$

Theorem 5.1 guarantees the existence of $D_{i, j}(\alpha)$ satisfying (6.1). Furthermore, as in Section 7 in [2], with the help of Proposition 5.3, we can prove that

$$
\inf _{u \in \mathcal{C}_{0}} \sup _{\alpha \in[0,1]} \|\left\langle W_{i}+\sum_{j} D_{i, j}(\alpha)\left[\eta\left(e_{j}\right)-\eta(0)\right]-L u \|_{\alpha}=0 .\right.
$$

Also, as in Lemma 5.2 of [5], we can prove that

$$
\inf _{u \in \mathcal{C}_{0}} \sup _{\alpha \in[0,1]} \|\left\langle W_{i}^{*}+\sum_{j} D_{i, j}(\alpha)\left[\eta\left(e_{j}\right)-\eta(0)\right]-L^{*} u \|_{\alpha}=0,\right.
$$

where $W_{i}^{*}$ is obtained replacing $p(y)$ by $p(-y)$ in formula (6.2).

To get an explicit formula for the diffusion coefficient, go back to Theorem 5.1 to get that

$$
D_{i, j}(\alpha)=\frac{-1}{\chi(\alpha)}\left\langle\left\langle\pi_{1} W_{i}\right\rangle_{\alpha, j}+\lim _{\lambda \rightarrow 0}\left\langle\left\langle\tilde{f}_{\lambda}^{i}, \mathfrak{m}_{j}\right\rangle_{0,0},\right.\right.
$$

where $\mathfrak{m}_{j}$ is given in (5.3) and where $\tilde{\mathfrak{f}}_{\lambda}^{i}$ is the solution of the resolvent equation

$$
\lambda \tilde{\mathfrak{f}}_{\lambda}^{i}-\mathfrak{L}_{\alpha} \tilde{\mathfrak{f}}_{\lambda}^{i}=-\mathfrak{T W}_{i} .
$$

Elementary computations give that 


$$
\begin{aligned}
& \mathfrak{T W}_{i}=-\chi(\alpha) \mathfrak{m}_{i}, \\
& \left\langle\left\langle\pi_{1} W_{i}\right\rangle_{\alpha, j}=-\frac{1}{2} \chi(\alpha) \sum y_{i} y_{j} p(y)=-\frac{1}{2} \chi(\alpha) \sigma_{i, j},\right.
\end{aligned}
$$

so that

$$
D_{i, j}(\alpha)=\frac{1}{2} \sigma_{i, j}+\lim _{\lambda \rightarrow 0} \chi(\alpha)\left\langle\left\langle\frac{\tilde{\mathfrak{f}}_{\lambda}^{i}}{\chi(\alpha)}, \mathfrak{m}_{j} \|_{0,0} .\right.\right.
$$

Let $\mathfrak{f}_{\lambda}^{i}=\tilde{\mathfrak{f}}_{\lambda}^{i} / \chi(\alpha)$ to obtain that $\mathfrak{f}_{\lambda}^{i}$ is the solution of the resolvent equation

$$
\lambda \mathfrak{f}_{\lambda}^{i}-\mathfrak{L}_{\alpha} \mathfrak{f}_{\lambda}^{i}=\mathfrak{m}_{i}
$$

and that

$$
D_{i, j}(\alpha)=\frac{1}{2} \sigma_{i, j}+\lim _{\lambda \rightarrow 0} \chi(\alpha)\left\langle f_{\lambda}^{i}, \mathfrak{m}_{j}\right\rangle_{0,0} .
$$

Remark 6.1. As we observed in the proof of Theorem 4.7, there exists $\mathfrak{f}^{i}$ in $\mathfrak{H}_{1,0}$ such that $\mathfrak{f}_{\lambda}^{i}$ converges to $\mathfrak{f}_{i}$, as $\lambda \downarrow 0$. As in Lemma 2.8 in [6], we get that $\lambda\left\langle\left\langle\mathfrak{f}_{\lambda}^{i}, f_{\lambda}^{i}\right\rangle_{0,0}\right.$ converges to zero, as $\lambda \downarrow 0$. This allows us to prove that $D(\alpha) \geqslant 1 / 2 \sigma$ in the sense of matrix, since

$$
\lim _{\lambda \rightarrow 0} \sum a_{i}\left\langle\mathfrak{f}_{\lambda}^{i}, \mathfrak{m}_{j}\right\rangle_{0,0} a_{j}=\left\|\sum a_{i} \mathfrak{f}^{i}\right\|_{1,0}^{2} \geqslant 0 .
$$

Proof of Theorem 2.1. Considering the formula presented in (6.6) for the diffusion coefficient of the hydrodynamic equation, the proof of its regularities properties on $(0,1)$ is a simple consequence of the following lemma. At the end of this section we give a reference to understand the behavior at the boundary.

Lemma 6.2. Take $\mathfrak{r}$ and $\mathfrak{S}$, finite supported functions in $\mathcal{F}_{*}$ with values not depending on $\alpha$. Consider the resolvent equation

$$
\lambda \mathfrak{f}_{\lambda}(\alpha)-\mathfrak{L}_{\alpha} \mathfrak{f}_{\lambda}(\alpha)=\mathfrak{r} .
$$

For each $\lambda>0$, consider the function $\mathfrak{G}_{\lambda}:[0,1] \rightarrow \mathbb{R}$ defined by

$$
\mathfrak{G}_{\lambda}(\alpha)=\left\langle\mathfrak{f}_{\lambda}(\alpha), \mathfrak{S}\right\rangle_{0,0} .
$$

Then, there exists a subsequence $\lambda_{k} \downarrow 0$ such that $\mathfrak{G}_{\lambda_{k}}$ converges uniformly to a smooth function on $[0,1]$. Furthermore, the limit is continuous in the whole interval and $C^{\infty}$ in its interior.

Proof. To prove the existence of such subsequence we will show that the functions $\mathfrak{G}_{\lambda}$ are smooth for each $\lambda>0$ and we will get uniform bounds, in $\lambda>0$, for the $L^{\infty}$ norm of the derivatives:

$$
\sup _{\lambda} \sup _{\alpha \in[\varepsilon, 1-\varepsilon]}\left|\mathfrak{G}_{\lambda}^{k}(\alpha)\right| \leqslant A_{k} \quad \forall \varepsilon, \forall k,
$$

where the upper index indicate the $k$ th derivate. For $k=0$ we need to show that the functions $\mathfrak{G}_{\lambda}$ are continuous and uniformly bounded in $[0,1]$. In order to get the announced bound for $k=0$ bound, take inner product $\langle\cdot, \cdot\rangle_{0,0}$ with $\mathfrak{f}_{\lambda}$ on both sides of Eq. (6.7) and use that $\left|\langle\mathfrak{u}, \mathfrak{v}\rangle_{0,0}\right| \leqslant\|\mathfrak{u}\|_{1,0}\|\mathfrak{v}\|_{-1,0}$ to get that

$$
\begin{aligned}
& \left\langle\lambda \mathfrak{f}_{\lambda}(\alpha), \mathfrak{f}_{\lambda}(\alpha)\right\rangle_{0,0}-\left\langle\mathfrak{L}_{\alpha} \mathfrak{f}_{\lambda}(\alpha), \mathfrak{f}_{\lambda}(\alpha) \|_{0,0}=\left\langle\left\langle\mathfrak{r}, \mathfrak{f}_{\lambda}(\alpha) \|_{0,0},\right.\right.\right. \\
& \lambda\left\|\mathfrak{f}_{\lambda}(\alpha)\right\|_{0,0}^{2}+\left\|\mathfrak{f}_{\lambda}(\alpha)\right\|_{1,0}^{2} \leqslant\left\|\mathfrak{f}_{\lambda}(\alpha)\right\|_{1,0}\|\mathfrak{r}\|_{-1,0}, \\
& \left\|\mathfrak{f}_{\lambda}(\alpha)\right\|_{1,0} \leqslant\|\mathfrak{r}\|_{-1,0} .
\end{aligned}
$$


Then, for every $\lambda>0$, we have that

$$
\left|\mathfrak{G}_{\lambda}(\alpha)\right|=\mid\left\langle\mathfrak{f}_{\lambda}(\alpha), \mathfrak{S}\left\|_{0,0} \mid \leqslant\right\| \mathfrak{r}\left\|_{-1,0}\right\| \mathfrak{S} \|_{-1,0},\right.
$$

where, by hypothesis, the last term does not depend on $\alpha$.

The following step is to differentiate (and also prove continuity on the whole interval $[0,1]$ ). This is the content of Lemma 6.3, below. It says that we can differentiate $\mathfrak{f}_{\lambda}(\alpha)$ in $\|\cdot\|_{1, k}$. Furthermore: the derivate, $\mathfrak{f}_{\lambda}^{\prime}(\alpha)$ satisfies the resolvent equation

$$
\lambda \mathfrak{f}_{\lambda}^{\prime}(\alpha)-\mathfrak{L}_{\alpha} \mathfrak{f}_{\lambda}^{\prime}(\alpha)=\mathfrak{L}^{\prime}(\alpha) \mathfrak{f}_{\lambda}(\alpha)
$$

with $\mathfrak{L}^{\prime}(\alpha)$ defined below (formula (6.13)). Then $\mathfrak{G}_{\lambda}^{\prime}(\alpha)=\left\langle\mathfrak{f}_{\lambda}^{\prime}(\alpha), \mathfrak{S}\right\rangle_{0,0}$. Once we have differentiated, we need to bound. For that, recall Proposition 4.8. and Corollary 4.3 to get that

$$
\left\|\mathfrak{g}_{\lambda}^{\prime}(\alpha)\right\|_{1,0} \leqslant\left\|\mathfrak{L}^{\prime}(\alpha) \mathfrak{f}_{\lambda}(\alpha)\right\|_{-1,0} \leqslant C(\alpha)\left\|\mathfrak{f}_{\lambda}(\alpha)\right\|_{1,0} .
$$

Collecting all these estimates, we get that

$$
\left|\mathfrak{G}_{\lambda}^{\prime}(\alpha)\right|=\mid\left\langle\mathfrak{f}_{\lambda}^{\prime}(\alpha), \mathfrak{S}\left\|_{0,0} \mid \leqslant\right\| \mathfrak{f}_{\lambda}^{\prime}(\alpha)\left\|_{1,0}\right\| \mathfrak{S}\left\|_{-1,0} \leqslant C(\alpha)\right\| \mathfrak{r}\left\|_{-1,0}\right\| \mathfrak{S} \|_{-1,0}\right.
$$

for $C(\alpha)$ continuous in $(0,1)$. Now, applying to Corollary 4.3 , we can check that $\mathfrak{L}^{\prime}(\alpha) \mathfrak{f}_{\lambda}(\alpha)$ satisfies the hypothesis of Lemma 6.3. So, iterating the previous argument, we can differentiate and bound.

We end this section with the announced result that gives sense to differentiate.

Lemma 6.3. Consider $\mathfrak{g}(\alpha)$ with $\|\mathfrak{g}(\alpha)\|_{-1, k}<\infty$ for every $k$. For each $\lambda>0$, let $\mathfrak{f}_{\lambda}(\alpha)$ be the solution of the resolvent equation

$$
\lambda \mathfrak{f}_{\lambda}(\alpha)-\mathfrak{L}_{\alpha} \mathfrak{f}_{\lambda}(\alpha)=\mathfrak{g}(\alpha) .
$$

Fix $\alpha \in(0,1)$. If

$$
\lim _{h \rightarrow 0}\|\mathfrak{g}(\alpha+h)-\mathfrak{g}(\alpha)\|_{-1, k}=0
$$

for all $k \geqslant 0$, then we get that

$$
\begin{aligned}
& \lim _{h \rightarrow 0}\left\|\mathfrak{f}_{\lambda}(\alpha+h)-\mathfrak{f}_{\lambda}(\alpha)\right\|_{1, k} \rightarrow 0, \\
& \lim _{h \rightarrow 0}\left\|\mathfrak{f}_{\lambda}(\alpha+h)-\mathfrak{f}_{\lambda}(\alpha)\right\|_{0, k} \rightarrow 0
\end{aligned}
$$

for all $k$. Furthermore: suppose that there exists a function $\mathfrak{G}(\alpha)$, with $\|\mathfrak{G}(\alpha)\|_{-1, k}<\infty$ for every $k$, such that

$$
\left\|\frac{\mathfrak{g}(\alpha+h)-\mathfrak{g}(\alpha)}{h}-\mathfrak{G}(\alpha)\right\|_{-1, k} \rightarrow 0
$$

as $h \rightarrow 0$ for every $k$. Then, for $\alpha \in(0,1)$ and $\lambda$ fixed, there exist $\mathfrak{F}_{\lambda}(\alpha)$ solution of the resolvent equation

$$
\lambda \mathfrak{F}_{\lambda}(\alpha)-\mathfrak{L}_{\alpha} \mathfrak{F}_{\lambda}(\alpha)=\mathfrak{G}(\alpha)+\mathfrak{L}_{\alpha}^{\prime} \mathfrak{f}_{\lambda}(\alpha),
$$

where

$$
\mathfrak{L}_{\alpha}^{\prime}=-2 \mathfrak{L}_{d}+\sqrt{\chi(\alpha)}^{\prime}\left\{\mathfrak{L}_{+}+\mathfrak{L}_{-}\right\},
$$

such that

$$
\begin{aligned}
& \left\|\frac{\mathfrak{f}_{\lambda}(\alpha+h)-\mathfrak{f}_{\lambda}(\alpha)}{h}-\mathfrak{F}_{\lambda}(\alpha)\right\|_{1, k} \rightarrow 0, \\
& \left\|\frac{\mathfrak{f}_{\lambda}(\alpha+h)-\mathfrak{f}_{\lambda}(\alpha)}{h}-\mathfrak{F}_{\lambda}(\alpha)\right\|_{0, k} \rightarrow 0
\end{aligned}
$$

as $h \rightarrow 0$, for every $k$. 
Proof. We have fixed $\alpha \in(0,1)$ and $\lambda>0$. Call $R(\alpha)=\sqrt{\chi(\alpha)}$. By Proposition 4.8, in order to guarantee existence of solution for Eq. (6.12), we need to prove that $\left\|\mathfrak{G}(\alpha)+\mathfrak{L}_{\alpha}^{\prime} \mathfrak{f}_{\lambda}(\alpha)\right\|_{-1,1}<\infty$. By hypothesis, $\|\mathfrak{G}(\alpha)\|_{-1,1}<\infty$. For the other term, use Corollary 4.3 and Lemma 4.10, for each of the following inequalities

$$
\left\|\mathfrak{L}_{*} \mathfrak{f}_{\lambda}(\alpha)\right\|_{-1,1} \leqslant C_{0}\left\|\mathfrak{f}_{\lambda}(\alpha)\right\|_{1,1} \leqslant C_{1}\|\mathfrak{g}(\alpha)\|_{-1,1},
$$

where the operator $\mathfrak{L}_{*}$ stands for $\mathfrak{L}_{s}, \mathfrak{L}_{d}, \mathfrak{L}_{+}, \mathfrak{L}_{-}$. Then, we get that

$$
\left\|\mathfrak{L}_{\alpha}^{\prime} \mathfrak{f}_{\lambda}(\alpha)\right\|_{-1,1} \leqslant C(\alpha)\|\mathfrak{g}(\alpha)\|_{-1,1} .
$$

Let $\mathfrak{F}_{\lambda}(\alpha)$ be the unique solution of (6.12). We want to see that the incremental quotient of $\mathfrak{f}_{\lambda}(\alpha)$ (in $\alpha$ ) converges to $\mathfrak{F}_{\lambda}(\alpha)$.

Consider the following resolvent equations:

$$
\begin{aligned}
& \lambda \mathfrak{f}_{\lambda}(\alpha)-\mathfrak{L}_{\alpha} \mathfrak{f}_{\lambda}(\alpha)=\mathfrak{g}(\alpha), \\
& \lambda \mathfrak{f}_{\lambda}(\alpha+h)-\mathfrak{L}_{(\alpha+h)} \mathfrak{f}_{\lambda}(\alpha+h)=\mathfrak{g}(\alpha+h) .
\end{aligned}
$$

Subtracting them we get that

$$
\begin{aligned}
\lambda\left[\mathfrak{f}_{\lambda}(\alpha+h)-\mathfrak{f}_{\lambda}(\alpha)\right]-\mathfrak{L}_{\alpha}\left[\mathfrak{f}_{\lambda}(\alpha+h)-\mathfrak{f}_{\lambda}(\alpha)\right]= & \mathfrak{g}(\alpha+h)-\mathfrak{g}(\alpha)-2 h \mathfrak{L}_{d} \mathfrak{f}_{\lambda}(\alpha+h) \\
& +(R(\alpha+h)-R(\alpha))\left(\mathfrak{L}_{+}+\mathfrak{L}_{-}\right) \mathfrak{f}_{\lambda}(\alpha+h) .
\end{aligned}
$$

At this point, using the bounds obtained in Proposition 4.8 and computing the $\|\cdot\|_{-1, k}$ norm of the right hand side of (6.14), we get the convergence in (6.9).

Consider the following objects

$$
\begin{aligned}
& \mathfrak{f}_{\lambda}^{*}(\alpha, h)=\frac{\mathfrak{f}_{\lambda}(\alpha+h)-\mathfrak{f}_{\lambda}(\alpha)}{h}-\mathfrak{F}_{\lambda}(\alpha), \\
& \mathfrak{g}^{*}(\alpha, h)=\frac{\mathfrak{g}(\alpha+h)-\mathfrak{g}(\alpha)}{h}-\mathfrak{G}(\alpha), \\
& R^{*}(\alpha, h)=\frac{R(\alpha+h)-R(\alpha)}{h}-R^{\prime}(\alpha) .
\end{aligned}
$$

Subtract Eq. (6.12) from Eq. (6.14) divided by $h$ to get that

$$
\begin{aligned}
\lambda \mathfrak{f}_{\lambda}^{*}(\alpha, h)-\mathfrak{L}_{\alpha} \mathfrak{f}_{\lambda}^{*}(\alpha, h)= & \mathfrak{g}^{*}(\alpha, h)-2 \mathfrak{L}_{d}\left[\mathfrak{f}_{\lambda}(\alpha+h)-\mathfrak{f}_{\lambda}(\alpha)\right]+R^{*}(\alpha, h)\left(\mathfrak{L}_{+}+\mathfrak{L}_{-}\right) \mathfrak{f}_{\lambda}(\alpha+h) \\
& +R^{\prime}(\alpha)\left(\mathfrak{L}_{+}+\mathfrak{L}_{-}\right)\left[\mathfrak{f}_{\lambda}(\alpha+h)-\mathfrak{f}_{\lambda}(\alpha)\right] .
\end{aligned}
$$

Using the hypothesis concerning $\mathfrak{g}(\alpha)$ and $\mathfrak{G}(\alpha)$, Lemma 4.10 and Corollary 4.3 , we can see that the $\|\cdot\|_{-1, k}$ norm of each term on the right hand side of the previous expression vanishes as $h \downarrow 0$. Then, applying Lemma 4.10 we conclude the result.

Remark 6.4. In order to prove differentiability of the functions $\left\{D_{i, j}(\alpha), 1 \leqslant i, j \leqslant d\right\}$ at the boundary of the interval $[0,1]$, we change the parametrization with $\alpha=\sin ^{2}(t)$, with $t \in[0, \pi / 2]$, and reproduce the work done in [8].

\section{Acknowledgements}

The author wishes to thank her thesis advisor Claudio Landim, for suggesting the problem and valuable discussions. 


\section{Appendix A. Decomposition of a mean zero probability as a convex combination of loop probabilities}

We start explaining what a loop probability is. Given $a_{1}, \ldots, a_{N}$ points in $\mathbb{Z}_{*}^{d}$ such that $\sum_{i=1}^{N} a_{i}=0$, consider the probability $\pi$ that assigns mass $1 / N$ over each $a_{i}$, for $i=1, \ldots, N$. As we do not require the points $a_{i}$ to be different, this is not necessarily an uniform probability. Observe that $\pi$ is a mean zero probability; we call it a loop probability. In order to motivate the name of this probability, set $y_{i}=\sum_{j=1}^{i} a_{j}$ and observe that $y_{N}=0$. This means that starting from the origin, jumping from $y_{i}$ to $y_{i+1}$ we arrive back to the origin. The points $y_{0}=0, y_{1}, \ldots, y_{N}$ form a loop (or cycle). $y_{i+1}-y_{i}=a_{i}$ is called a jump.

Definition A.1. A probability $\pi$ is a loop probability if there exists a closed path $y_{0}=0, y_{1}, \ldots, y_{N}=0$ in $\mathbb{Z}^{d}$ with $y_{i+1} \neq y_{i}$ such that

$$
\pi(x)=\frac{1}{N} \sum_{i=0}^{N-1} I_{\left\{x=y_{i+1}-y_{i}\right\}} .
$$

Definition A.2. Given a probability $p$ on $\mathbb{Z}^{d}$, we say that $x$ is in $\mathbb{S}_{p}$, the support of $p$, if $p(x)>0$ :

$$
\mathbb{S}_{p}=\left\{x \in \mathbb{Z}^{d} ; p(x)>0\right\} .
$$

We say that $p$ is compactly supported if $\mathbb{S}_{p}$ is finite.

The main object of this appendix is to prove that every compactly supported mean zero probability $p$ on $\mathbb{Z}_{*}^{d}$ may be written as a convex combination of loop probabilities.

Lemma A.3. Given a mean zero compactly supported probability $p$ on $\mathbb{Z}_{*}^{d}$, there exists $\alpha_{j}>0$ for $j=1, \ldots, s$ with $\sum_{j=1}^{s} \alpha_{j}=1$ and loop probabilities $\pi_{j}$ supported in $\mathbb{Z}_{*}^{d}$ such that

$$
p=\sum_{j=1}^{s} \alpha_{j} \pi_{j}
$$

Proof. We will use $z_{j}$ to denote vectors in different spaces and $z_{j}^{i}$ for the $i$ th coordinate of the vector $z_{j}$. Observe that if $p\left(x_{l}\right) \in \mathbb{Q}$ for all $x_{l} \in \mathbb{S}_{p}$, then $p$ is itself a loop probability. In this case we get that $p\left(x_{l}\right)=m_{l} / b$ for all $x_{l} \in \mathbb{S}_{p}$, with $m_{l}, b \in \mathbb{N}-\{0\}$ and $\sum m_{l}=b$. This corresponds to a loop probability taking the jump $x_{l} m_{l}$ times.

We will prove the lemma by induction in $n$, the number of points in the support of the probability $p: n=\# \mathbb{S}_{p}$. Observe that, since the probability is defined on $\mathbb{Z}_{*}^{d}$ and has mean zero, the support has at least two elements and $0 \notin \mathbb{S}_{p}$. Because of that, we start the induction with $n=2$.

(1) $n=2$. In this case we get that exist $x_{1}, x_{2} \in \mathbb{Z}_{*}^{d}$ such

$$
x_{1} p\left(x_{1}\right)+x_{2} p\left(x_{2}\right)=0 \quad \text { and } \quad p\left(x_{1}\right)+p\left(x_{2}\right)=1 .
$$

This two equations determine $p$. Solving for $p\left(x_{1}\right), p\left(x_{2}\right)$, we get that

$$
p\left(x_{1}\right)=-x_{2}^{i} /\left(x_{1}^{i}-x_{2}^{i}\right) \quad \text { and } \quad p\left(x_{2}\right)=x_{1}^{i} /\left(x_{1}^{i}-x_{2}^{i}\right),
$$

independently of $i$. This shows that the probability $p$ takes values in $\mathbb{Q}$ and then it is a loop probability.

(2) Inductive step: consider $p$ supported in a set of cardinality $n>2$. We would like to write

$$
p=c_{1} \pi_{1}+\left(1-c_{1}\right) \tilde{p}
$$

for some $0<c_{1}<1, \pi_{1}$ a loop probability, $\tilde{p}$ a mean zero probability on $\mathbb{Z}_{*}^{d}$, supported in a set with less than $n$ points. In this case, by the inductive hypothesis, we will be able to decompose $\tilde{p}$ as a convex combination of loop probabilities and therefore, the same holds for $p$. 
The problem is reduced to prove the existence of $\pi_{1}$, a loop probability concentrated in $\mathbb{S}_{p}=\left\{x_{1}, \ldots, x_{n}\right\}$. If such $\pi_{1}$ exists, take

$$
c_{1}=\min _{i} p\left(x_{i}\right) / \pi_{1}\left(x_{i}\right) .
$$

Since both $p$ and $\pi_{1}$ are probabilities, $c_{1} \leqslant 1$. If $c_{1}=1$ then $p=\pi_{1}$ and $p$ is a loop probability. Otherwise, $\tilde{p}$ defined by

$$
\tilde{p}\left(x_{i}\right)=\frac{p\left(x_{i}\right)-c_{1} \pi_{1}\left(x_{i}\right)}{1-c_{1}}
$$

is a mean zero probability on $\mathbb{Z}_{*}^{d}$ whose support is smaller than the support of $p$, as we wanted.

We will now prove the existence of a loop probability concentrated in $x_{1}, \ldots, x_{n}$. We are looking for a linear combination of $x_{i}$ with rational positive coefficients that adds up to zero, i.e., $q_{i} \in \mathbb{Q}, q_{i}>0$ such that $\sum q_{i} x_{i}=0$. Then, we normalize and obtain the desired probability.

Since $\sum_{i=1}^{n} p\left(x_{i}\right) x_{i}=0$ with $p\left(x_{i}\right)>0$ for $i=1, \ldots, n$, we know that the vectors $x_{1}, \ldots, x_{n}$ are linearly dependent in $\mathbb{R}^{d}$. Without loss of generality, we may suppose that $\left\{x_{1}, \ldots, x_{s}\right\}$ is a basis of $V$, the linear subspace in $\mathbb{R}^{d}$ generated by $x_{1}, \ldots, x_{n}$, with $s<n$. Let $A$ be the $d \times s$ matrix defined by $A_{i, j}=x_{i}^{j}$. Observe that $A: \mathbb{R}^{s} \rightarrow V$ is one to one and that it inverse assignees to each vector $v \in V$ its coordinates in base $\left\{x_{1}, \ldots, x_{s}\right\}$. Consider the function $f: \mathbb{R}^{n-s} \rightarrow \mathbb{R}^{s}$, given by

$$
f\left(u_{1}, \ldots, u_{n-s}\right):=-A^{-1}\left(u_{1} x_{s+1}+u_{2} x_{s+2}+\cdots+u_{n-s} x_{n}\right) .
$$

Note that

$$
\sum_{i=1}^{n} u_{i} x_{i}=0 \quad \Leftrightarrow \quad\left(u_{1}, \ldots, u_{s}\right)=f\left(u_{s+1}, \ldots, u_{n}\right) .
$$

In particular, since $\sum_{i=1}^{n} p\left(x_{i}\right) x_{i}=0$, we get that

$$
p\left(x_{1}\right), \ldots, p\left(x_{s}\right)=f\left(p\left(x_{s+1}\right), \ldots, p\left(x_{n}\right)\right),
$$

with all the entries of $p\left(x_{i}\right)$ for $1 \leqslant i \leqslant n$ positive. By continuity of the linear transformation $f$, we can choose $\left(q_{s+1}, \ldots, q_{n}\right)$ close to $\left(p\left(x_{s+1}\right), \ldots, p\left(x_{n}\right)\right)$, positive and rational, such that $f\left(q_{s+1}, \ldots, q_{n}\right)$ is also positive (and clearly it is rational because $A^{-1}$ is a rational matrix). Then

$$
\tilde{u}_{1}, \ldots, \tilde{u}_{s}=f\left(q_{s+1}, \ldots, q_{n}\right) \quad \text { and } \quad \tilde{u}_{s+i}=q_{s+i}, \quad \text { for } i=1, \ldots, n-s
$$

are the coefficient that we are looking for.

\section{Appendix B. Estimates on the operators $\mathfrak{L}_{d}, \mathfrak{L}_{+}$and $\mathfrak{L}_{-}$}

In this appendix we prove some results involving the operators $\mathfrak{L}_{s}, \mathfrak{L}_{d}, \mathfrak{L}_{+}$and $\mathfrak{L}_{-}$. Most of them were presented in Section 4.

Recall that for $\mathfrak{f} \in \mathcal{F}_{*} \mathfrak{f}\left(S_{z} A\right)=\mathfrak{f}(A)$ for all $z$ in $A$.

A simple computation shows that the operators $\mathfrak{L}_{s}$ and $\mathfrak{L}_{d}$ send $\mathcal{I}_{*, n}$ into them self, while $\mathfrak{L}_{+}$and $\mathfrak{L}_{-}$map $\mathcal{I}_{*, n}$ into $\mathcal{I}_{*, n+1}$ and $\mathcal{I}_{*, n-1}$, respectively.

The following identity illustrates the fact that the space $\mathcal{I}_{*}$ enjoys some special properties. For every $\mathfrak{f}: \mathcal{E}_{*, 1} \rightarrow \mathbb{R}$,

$$
\left(\mathfrak{L}_{-} \mathfrak{f}\right)(\phi)=-2 \sum_{x \neq 0} a(x) \mathfrak{f}(\{x\}) .
$$


In particular, $\left(\mathfrak{L}_{-} \mathfrak{f}\right)(\phi)=0$ for all $\mathfrak{f}$ in $\mathcal{I}_{*, 1}$ because in this space $\mathfrak{f}(\{x\})=\mathfrak{f}(\{-x\})$ and $a(\cdot)$ is anti-symmetric. In contrast, $\left(\mathfrak{L}_{+} \mathfrak{g}\right)(\{x\})=0$ for all functions $\mathfrak{g}: \mathcal{E}_{*, 0} \rightarrow \mathbb{R}$ so that, for all $\mathfrak{f}$ in $\mathcal{I}_{*, 1}$ and all $\mathfrak{g}: \mathcal{E}_{*, 0} \rightarrow \mathbb{R}$,

$$
\mathfrak{L}_{-} \mathfrak{f}=0, \quad \mathfrak{L}_{+} \mathfrak{g}=0 .
$$

Other important consequences of working on the space $\mathcal{I}_{*}$ are stated in the following lemma.

Lemma B.1. For every $n \geqslant 1$ and every finitely supported functions $\mathfrak{u}, \mathfrak{v} \in \mathcal{F}_{*, n}$

$$
\left\langle\mathfrak{L}_{d} \mathfrak{u}, \mathfrak{v}\right\rangle=-\left\langle\mathfrak{u}, \mathfrak{L}_{d} \mathfrak{v}\right\rangle .
$$

For every finitely supported functions $\mathfrak{f}, \mathfrak{g}$ in $\mathcal{F}_{*, n-1}, \mathcal{F}_{*, n}$, respectively,

$$
\frac{1}{n+1}\left\langle\mathfrak{L}_{+} \mathfrak{f}, \mathfrak{g}\right\rangle=-\frac{1}{n}\left\langle\mathfrak{f}, \mathfrak{L}_{-} \mathfrak{g}\right\rangle .
$$

Proof. The first identity relies on the fact that $\sum_{x, y \in A} a(y-x)=0$. Note, however, that both pieces of the operator are needed.

For the second statement, observe that for each fixed density $\alpha$, given $f$ and $g$ in $\mathcal{C}_{0}$, we have that

$$
\left\langle L^{s} f, g\right\rangle=\left\langle\left\langle f, L^{s} g\right\rangle \quad \text { and } \quad\left\langle L^{a} f, g\right\rangle=-\left\langle\left\langle f, L^{a} g\right\rangle\right\rangle .\right.
$$

The second identity implies that

$$
\left\langle\left(\mathfrak{L}_{\alpha}-\mathfrak{L}^{s}\right) \mathfrak{f}, \mathfrak{g}\right\rangle_{0,0}=-\left\langle\left\langle\mathfrak{f},\left(\mathfrak{L}_{\alpha}-\mathfrak{L}^{s}\right) \mathfrak{g}\right\rangle_{0,0},\right.
$$

for $\mathfrak{f}, \mathfrak{g} \in \mathcal{F}_{*}$. Considering the first identity of the present lemma and choosing $\mathfrak{f} \in \mathcal{F}_{*, n-1}$, and $\mathfrak{g} \in \mathcal{F}_{*, n}$ get that

$$
\frac{1}{n+1}\left\langle\mathfrak{L}_{+} \mathfrak{f}, \mathfrak{g}\right\rangle=-\frac{1}{n}\left\langle\mathfrak{f}, \mathfrak{L}_{-} \mathfrak{g}\right\rangle
$$

Corollary B.2. The operators $\mathfrak{L}_{+}+\mathfrak{L}_{-}$and $\mathfrak{L}_{d}$ are anti-symmetric with respect to the inner product $\langle\cdot, \cdot\rangle_{0,0}$ :

$$
\begin{aligned}
& \left\langle\mathfrak{f},\left(\mathfrak{L}_{+}+\mathfrak{L}_{-}\right) \mathfrak{g}\right\rangle_{0,0}=-\left\langle\left(\left(\mathfrak{L}_{+}+\mathfrak{L}_{-}\right) \mathfrak{f}, \mathfrak{g}\right\rangle_{0,0},\right. \\
& \left\langle\mathfrak{f}, \mathfrak{L}_{d} \mathfrak{g}\right\rangle_{0,0}=-\left\langle\left\langle\mathfrak{L}_{d} \mathfrak{f}, \mathfrak{g}\right\rangle_{0,0}\right.
\end{aligned}
$$

for all finitely supported functions $\mathfrak{f}, \mathfrak{g}$ in $\mathcal{F}_{*}$. The same statement remains in force if $\mathfrak{L}_{+}+\mathfrak{L}_{-}$and $\mathfrak{L}_{d}$ are replaced by $\Pi_{n}\left(\mathfrak{L}_{+}+\mathfrak{L}_{-}\right) \Pi_{n}$ and $\Pi_{n} \mathfrak{L}_{d} \Pi_{n}$, respectively, for every $n \geqslant 1$ with $\mathfrak{f}$ and $\mathfrak{g}$ in $\mathcal{J}_{n}$, defined in (4.4).

Remark B.3. From the previous corollary, we get that, for every $\mathfrak{f}$ in $\mathcal{F}_{*},\left\langle\left\langle\mathfrak{L}_{\alpha} \mathfrak{f}, \mathfrak{f}\right\rangle_{0,0}=\left\langle\left\langle\mathfrak{L}_{\mathfrak{s}} \mathfrak{f}, \mathfrak{f}\right\rangle_{0,0}\right.\right.$. Consider $k \geqslant 1$. Given $\mathfrak{f} \in \mathbb{I}_{*}^{k}$, take $\mathfrak{f}_{n} \in \mathcal{F}_{*}$ such that $\left\|\mathfrak{f}_{n}-\mathfrak{f}\right\|_{0, k}$ goes to zero as $n \uparrow \infty$. Since $\mathfrak{L}_{\alpha}$ and $\mathfrak{L}_{s}$ are bounded operators from $\mathbb{I}_{*}^{k}$ to $\mathbb{I}_{*}^{0}$, we get that $\left\langle\left\langle\mathfrak{L}_{\alpha} \mathfrak{f}, \mathfrak{f}\right\rangle_{0,0}=\left\langle\mathfrak{L}_{s} \mathfrak{f}, \mathfrak{f}\right\rangle_{0,0}\right.$.

Recall Theorem 4.1 from Section 4. Hereafter the constant $C_{0}$ may change from line to line.

Theorem B.4. There exists a finite constant $C_{0}$, depending only on the probability $p$, such that

$$
\left\langle\mathfrak{L}_{d} \mathfrak{f}, \mathfrak{g}\right\rangle^{2} \leqslant C_{0}\left\langle\mathfrak{f},-\mathfrak{L}_{s} \mathfrak{f}\right\rangle\left\langle\mathfrak{g},-\mathfrak{L}_{s} \mathfrak{g}\right\rangle
$$

for each $\mathfrak{f}, \mathfrak{g}$ in $\mathcal{I}_{*, n}$. The same result remains in force if $\mathfrak{L}_{d}$ is replaced by $\mathfrak{L}_{+}$or $\mathfrak{L}_{-}$with $\mathfrak{g}$ in $\mathcal{I}_{*, n+1}$ and $\mathcal{I}_{*, n-1}$, respectively.

The proof of Theorem B.4 is divided in several lemmas. Before starting, we need to introduce some definitions and recall some results. Since the $\|\cdot\|_{1}$ norm plays a crucial role, we give its explicit form. For $\mathfrak{f} \in \mathcal{I}_{*, n}$, from the definition of the operator $\mathfrak{L}_{s}$ given in (3.18), we get that

$$
\left\langle\mathfrak{f},-\mathfrak{L}_{s} \mathfrak{f}\right\rangle=1 / 4 \sum_{x, y \in \mathbb{Z}_{*}^{d}} s(y-x) \sum_{B \in \mathcal{E}_{*, n}}\left[\mathfrak{f}\left(B_{x, y}\right)-\mathfrak{f}(B)\right]^{2}+1 / 2 \sum_{y} s(y) \sum_{B: y \notin B}\left[\mathfrak{f}\left(S_{y} B\right)-\mathfrak{f}(B)\right]^{2},
$$


where $B_{x, y}$ was defined in (3.16) and $S_{y} B$ in (3.7). Observe that first term of the previous expression, may be written as

$$
\frac{1}{2} \sum_{b \in \mathbb{Z}^{d}} s(b) \sum_{x \in \mathbb{Z}_{*}^{d}} \sum_{\substack{A \in \mathcal{E}_{*, n-1} \\ A \cap\{x, x+b\}=\emptyset}}[\mathfrak{f}(A \cup\{x+b\})-\mathfrak{f}(A \cup\{x\})]^{2} .
$$

Theorem B.4 will be proved for loop probabilities. Since we proved in Appendix A that every mean zero probability $p$ may be decomposed as a convex combination of loop probabilities, considering formula (B.3), there is no loss of generality in the proof of Theorem B.4 assuming that $p$ is a loop probability.

For a loop probability we prove Theorem B.4 by induction on the length of the loop. For the inductive step, we need to relate the $\|\cdot\|_{1}$ norms corresponding to different probabilities. For this purpose, we define the following objects. Recall that the support of the probability $p$ was defined as

$$
\mathbb{S}_{p}=\{x: p(x)>0\} .
$$

We say that $x$ is attainable if it may be connected with the origin in the following sense: there exists a sequence $z_{0}=0, z_{1}, \ldots, z_{n}=x$ with $p\left(z_{i+1}-z_{i}\right)>0$. We say that $x$ is attainable after $m$ steps if $m$ is the length of a shortest path connecting $x$ with the origin. We note by $\mathbb{A}_{p}$ the set of attainable points for the probability $p$. Observe that for mean zero probabilities, $\mathbb{A}_{p}=\mathbb{A}_{s}$ where $s$ is the symmetric part of the probability $p$. This result is clear for a loop probability and then, by the decomposition result, the same holds for every mean zero probability.

A straightforward computation considering that we are working in $\mathcal{I}_{*, n}$, shows that Dirichlet forms associated to different probabilities are related in the following way:

Remark B.5. Given two mean zero probabilities $p_{1}$ and $p_{2}$ with $\mathbb{S}_{p_{1}} \subseteq \mathbb{A}_{p_{2}}$, there exists a finite constant $C$ such that

$$
\left\langle\mathfrak{f},-\mathfrak{L}_{s}^{p_{1}} \mathfrak{f}\right\rangle \leqslant C\left\langle\mathfrak{f},-\mathfrak{L}_{s}^{p_{2}} \mathfrak{f}\right\rangle,
$$

where $\mathfrak{L}_{s}^{p_{i}}$ are the operators defined in (3.18), corresponding to the probabilities $p_{i}$ for $i=1,2$.

In what follows, we deal with the operators defined in (3.30), associated to different probabilities. In order to avoid confusions, we will use $\mathfrak{L}_{*}^{\pi}$ for $*=s, d,+,-$, to denote the corresponding operators related to the probability $\pi$. We are now able to start proving Theorem B.4. Almost all the computations are obtained performing some change of variables and considering that we are working with functions in the space $\mathcal{I}_{*}$.

Lemma B.6. Given a loop probability $\pi$ there exists a finite constant $C_{0}$, depending only on the probability $\pi$, such that

$$
\left\langle\mathfrak{g}, \mathfrak{L}_{+}^{\pi} \mathfrak{f}\right\rangle^{2} \leqslant C_{0}\left\langle\mathfrak{f},-\mathfrak{L}_{s}^{\pi} \mathfrak{f}\right\rangle\left\langle\mathfrak{g},-\mathfrak{L}_{s}^{\pi} \mathfrak{g}\right\rangle,
$$

for each $\mathfrak{f} \in \mathcal{I}_{*, n-1}, \mathfrak{g} \in \mathcal{I}_{*, n}$.

Proof. The proof of this result is by induction in the length of the loop. We show the inductive step and prove the result for a loop of length three, since for smaller loop the operator vanish. Note that the constants $C$ and $C_{0}$ may change from line to line.

Fix a loop probability $\pi=\left\{y_{0}, \ldots, y_{m}\right\}$, with $a_{i}=y_{i}-y_{i-1}$, for $i=1, \ldots, m$. An elementary computation shows that

$$
\begin{aligned}
\left\langle\mathfrak{g}, \mathfrak{L}_{+}^{\pi} \mathfrak{f}\right\rangle= & 2 \sum_{A \in \mathcal{E}_{*, n}} \sum_{x, y \in A} a(y-x) \mathfrak{g}(A) \mathfrak{f}(A \backslash\{y\}) \\
& +2 \sum_{A \in \mathcal{E}_{*, n}} \sum_{x \in A} a(x) \mathfrak{g}(A)\left[\mathfrak{f}(A \backslash\{x\})-\mathfrak{f}\left(S_{x}(A \backslash\{x\})\right)\right] .
\end{aligned}
$$


Observe that $a(y-x)= \pm 1 / 2 m$ for $y-x= \pm a_{i}$ and $a(y-x)=0$ otherwise. Then, except for $y-x=a_{i}$ or $y-x=-a_{i}, a(y-x)=0$. Thus, the first term in the right hand side of (B.6) is equal to

$$
\begin{aligned}
& \frac{1}{m} \sum_{i=1}^{m} \sum_{x \neq 0,-a_{i}} \sum_{\substack{A \in \mathcal{E}_{*, n-2} \\
A \cap\left\{x, x+a_{i}\right\}=\emptyset}} \mathfrak{f}(A \cup\{x\}) \mathfrak{g}\left(A \cup\left\{x, x+a_{i}\right\}\right) \\
& -\frac{1}{m} \sum_{i=1}^{m} \sum_{x \neq 0, a_{i}} \sum_{\substack{A \in \mathcal{E}_{*, n-2} \\
A \cap\left\{x, x-a_{i}\right\}=\emptyset}} \mathfrak{f}(A \cup\{x\}) \mathfrak{g}\left(A \cup\left\{x, x-a_{i}\right\}\right) .
\end{aligned}
$$

We perform a change of variables in the second term of the previous expression and get that the difference is equal to

$$
\frac{1}{m} \sum_{i=1}^{m} \sum_{x \neq 0,-a_{i}} \sum_{\substack{A \in \mathcal{E}_{*, n-2}-\\ A \cap\left\{x, x+a_{i}\right\}=\emptyset}}\left[\mathfrak{f}(A \cup\{x\})-\mathfrak{f}\left(A \cup\left\{x+a_{i}\right\}\right)\right] \mathfrak{g}\left(A \cup\left\{x, x+a_{i}\right\}\right) .
$$

Recall that $a(x)=1 / 2 m$ for $x=a_{i}, a(x)=-1 / 2 m$ for $x=-a_{i}$ and use this fact to write the second term on the right hand side of (B.6) as

$$
\begin{aligned}
& \frac{1}{m} \sum_{i=1}^{m} \sum_{\substack{A \in \mathcal{E}_{*, n-1} \\
A \cap\left\{a_{i}\right\}=\emptyset}}\left[\mathfrak{f}(A)-\mathfrak{f}\left(S_{a_{i}} A\right)\right] \mathfrak{g}\left(A \cup\left\{a_{i}\right\}\right)-\frac{1}{m} \sum_{i=1}^{m} \sum_{\substack{A \in \mathcal{E}_{*, n-1} \\
A \cap\left\{-a_{i}\right\}=\emptyset}}\left[\mathfrak{f}(A)-\mathfrak{f}\left(S_{-a_{i}} A\right)\right] \mathfrak{g}\left(A \cup\left\{-a_{i}\right\}\right) \\
& \quad=2 \frac{1}{m} \sum_{i=1}^{m} \sum_{\substack{A \in \mathcal{E}_{*, n-1} \\
A \cap\left\{a_{i}\right\}=\emptyset}}\left[\mathfrak{f}(A)-\mathfrak{f}\left(S_{a_{i}} A\right)\right] \mathfrak{g}\left(A \cup\left\{a_{i}\right\}\right),
\end{aligned}
$$

where we performed a change of variables in the second line $\left(B=S_{-a_{i}} B\right)$ and used that for $\mathfrak{g} \in \mathcal{I}_{*}$ we have that $\mathfrak{g}\left(S_{a_{i}} B \cup\left\{-a_{i}\right\}\right)=\mathfrak{g}\left(S_{a_{i}}\left(B \cup\left\{a_{i}\right\}\right)\right)=\mathfrak{g}\left(B \cup\left\{a_{i}\right\}\right)$ to obtain the last identity. Let

$$
\begin{aligned}
N_{b} & =\sum_{x \neq 0,-b} \sum_{\substack{A \in \mathcal{E}_{*, n-2} \\
A \cap\{x, x+b\}=\emptyset}}[\mathfrak{f}(A \cup\{x\})-\mathfrak{f}(A \cup\{x+b\})] \mathfrak{g}(A \cup\{x, x+b\}), \\
M_{b} & =\sum_{\substack{A \in \mathcal{E}_{*, n-1} \\
A \cap\{b\}=\emptyset}}\left[\mathfrak{f}(A)-\mathfrak{f}\left(S_{b} A\right)\right] \mathfrak{g}(A \cup\{b\}),
\end{aligned}
$$

so that

$$
\left\langle\mathfrak{g}, \mathfrak{L}_{+}^{\pi} \mathfrak{f}\right\rangle=\frac{1}{m} \sum_{i=1}^{m} N_{a_{i}}+\frac{2}{m} \sum_{i=1}^{m} M_{a_{i}} .
$$

We are ready to perform the inductive step. Let $z=\sum_{i=3}^{m} a_{i}$ and consider the loop probabilities $\pi_{1}=$ $\left\{y_{0}, y_{1}, y_{2}, y_{0}\right\}$ and $\pi_{2}=\left\{y_{0}, y_{2}, y_{3}, \ldots, y_{m}\right\}$ corresponding to jumps $a_{1}, a_{2}, z$ and $-z, a_{3}, \ldots, a_{m}$, respectively. Observing that $N_{-b}=-N_{b}$ and $M_{-b}=-M_{b}$ we get that

$$
\begin{aligned}
\left\langle\mathfrak{g}, \mathfrak{L}_{+}^{\pi} \mathfrak{f}\right\rangle & =1 / m\left[\left(N_{a_{1}}+N_{a_{2}}+N_{z}\right)+N_{-z}+\sum_{i=3}^{m} N_{a_{i}}\right]+1 / m\left[\left(M_{a_{1}}+M_{a_{2}}+M_{z}\right)+M_{-z}+\sum_{i=3}^{m} M_{a_{i}}\right] \\
& =3 / m\left\langle\mathfrak{g}, \mathfrak{L}_{+}^{\pi_{1}} \mathfrak{f}\right\rangle+m-1 / m\left\langle\mathfrak{g}, \mathfrak{L}_{+}^{\pi_{2}} \mathfrak{f}\right\rangle .
\end{aligned}
$$


Since $\mathbb{S}_{\pi_{i}} \subset \mathbb{A}_{\pi}$, by Remark B.5 we get that $\left\langle\mathfrak{f},-\mathfrak{L}_{s}^{\pi_{i}} \mathfrak{f}\right\rangle \leqslant C\left\langle\mathfrak{f},-\mathfrak{L}_{s}^{\pi} \mathfrak{f}\right\rangle$ for $i=1,2$. This fact and the inductive hypothesis give that

$$
\left\langle\mathfrak{g}, \mathfrak{L}_{+}^{\pi} \mathfrak{f}\right\rangle^{2} \leqslant C\left\{\left\langle\mathfrak{f}, \mathfrak{L}_{s}^{\pi_{1}} \mathfrak{f}\right\rangle\left\langle\mathfrak{g}, \mathfrak{L}_{s}^{\pi_{1}} \mathfrak{g}\right\rangle+\left\langle\mathfrak{f}, \mathfrak{L}_{s}^{\pi_{2}} \mathfrak{f}\right\rangle\left\langle\mathfrak{g}, \mathfrak{L}_{s}^{\pi_{2}} \mathfrak{g}\right\rangle\right\} \leqslant C\left\langle\mathfrak{f}, \mathfrak{L}_{s}^{\pi} \mathfrak{f}\right\rangle\left\langle\mathfrak{g}, \mathfrak{L}_{s}^{\pi} \mathfrak{g}\right\rangle,
$$

which concludes the inductive step.

Now, we need to prove the estimate for a loop probability of length three. Consider $\pi$ corresponding to jumps $a_{i}$ for $i=1,2,3$ with $a_{1}+a_{2}+a_{3}=0$. We start showing that $\left\{\sum_{i=1}^{3} N_{a_{i}}\right\}^{2} \leqslant C\left\langle\mathfrak{f}, \mathfrak{L}_{s}^{\pi} \mathfrak{f}\right\rangle\left\langle\mathfrak{g}, \mathfrak{L}_{s}^{\pi} \mathfrak{g}\right\rangle$ and then we prove the same kind of bound for $\left\{\sum_{i=1}^{3} M_{a_{i}}\right\}^{2}$. To keep notation simple, let $d=1$ and take $a_{1}=1, a_{2}=2$ and $a_{3}=-3$. According to definition (B.7) for $N_{b}$, we get that

$$
\begin{aligned}
\sum_{i=1}^{3} N_{a_{i}}= & \sum_{x \neq 0,-1} \sum_{\substack{A \in \mathcal{E}_{*, n-1} \\
A \cap\{x, x+1\}=\emptyset}}[\mathfrak{f}(A \cup\{x\})-\mathfrak{f}(A \cup\{x+1\})] \mathfrak{g}(A \cup\{x, x+1\}) \\
& +\sum_{x \neq-1,-3} \sum_{\substack{A \in \mathcal{E}_{*, n-1} \\
A \cap\{x+1, x+3\}=\emptyset}}[\mathfrak{f}(A \cup\{x+1\})-\mathfrak{f}(A \cup\{x+3\})] \mathfrak{g}(A \cup\{x+1, x+3\}) \\
& +\sum_{x \neq 0,-3} \sum_{\substack{A \in \mathcal{E}_{*, n-1} \\
A \cap\{x, x+3\}=\emptyset}}[\mathfrak{f}(A \cup\{x+3\})-\mathfrak{f}(A \cup\{x\})] \mathfrak{g}(A \cup\{x, x+3\}),
\end{aligned}
$$

where for the second and last line we performed a change of variable. We will decompose this sum in three terms: $\sum_{i=1}^{3} N_{a_{i}}=T_{1}+T_{2}+T_{3}$. The decomposition appears because we want to add over the same values of $x$ and then we work for adding in the same sets $A$. For $T_{1}$, take from (B.9) $x=-3$ in the first line, $x=0$ in the second line and $x=-1$ in the last one. $T_{2}$ is obtained taking $x \neq 0,-1,-3$ in the three sums of (B.9) and imposing $A$ to contain $x+3, x$ and $x+1$ in each line, respectively. Finally, $T_{3}$ is obtained taking $x \neq 0,-1,-3$ in all the sums and imposing $A$ not to contain each of the previous elements. Recall that we are working with finite sets $A$ in $\mathbb{Z}_{*}^{d}$. Sometimes we omit from the notation the cardinal of $A$. Some others we put in evidence that $A \cap\{0\}=\emptyset$. For $T_{1}$, we get

$$
\begin{aligned}
T_{1}= & \sum_{A \cap\{-3,-2,0\}=\emptyset}[\mathfrak{f}(A \cup\{-3\})-\mathfrak{f}(A \cup\{-2\})] \mathfrak{g}(A \cup\{-2,-3\}) \\
& +\sum_{A \cap\{0,1,3\}=\emptyset}[\mathfrak{f}(A \cup\{1\})-\mathfrak{f}(A \cup\{3\})] \mathfrak{g}(A \cup\{1,3\}) \\
& +\sum_{A \cap\{-1,0,2\}=\emptyset}[\mathfrak{f}(A \cup\{2\})-\mathfrak{f}(A \cup\{-1\})] \mathfrak{g}(A \cup\{-1,2\}) .
\end{aligned}
$$

Let $S_{3} A=B$ in the second line of (B.10) and $S_{2} A=B$ in the third one to get that

$$
\begin{aligned}
T_{1}= & \sum_{A \cap\{-3,-2,0\}=\emptyset}[\mathfrak{f}(A \cup\{-3\})-\mathfrak{f}(A \cup\{-2\})] \mathfrak{g}(A \cup\{-2,-3\}) \\
& +\sum_{A \cap\{-3,-2,0\}=\emptyset}\left[\mathfrak{f}\left(S_{-3} A \cup\{1\}\right)-\mathfrak{f}\left(S_{-3} A \cup\{3\}\right)\right] \mathfrak{g}\left(S_{-3} A \cup\{1,3\}\right) \\
& +\sum_{A \cap\{-3,-2,0\}=\emptyset}\left[\mathfrak{f}\left(S_{-2} A \cup\{2\}\right)-\mathfrak{f}\left(S_{-2} A \cup\{-1\}\right)\right] \mathfrak{g}\left(S_{-2} A \cup\{-1,2\}\right) .
\end{aligned}
$$

Recall that, by definition of $S_{z}, S_{-2} A \cup\{-1,2\}=S_{-2}(A \cup\{-2,-3\})$. In this case, since $\mathfrak{f}$ and $\mathfrak{g}$ belong to $\mathcal{I}_{*}$, we get that $\mathfrak{g}\left(S_{-2} A \cup\{-1,2\}\right)=\mathfrak{g}\left(S_{-2}(A \cup\{-2,-3\})\right)=\mathfrak{g}(A \cup\{-2,-3\})$. It is not difficult to check, using this kind of identities, that the previous expression vanishes. For $T_{2}$, we get that 


$$
\begin{aligned}
T_{2}= & \sum \sum[\mathfrak{f}(A \cup\{x x+3\})-\mathfrak{f}(A \cup\{x+1, x+3\})] \mathfrak{g}(A \cup\{x, x+1, x+3\}) \\
& +\sum \sum[\mathfrak{f}(A \cup\{x, x+1\})-\mathfrak{f}(A \cup\{x, x+3\})] \mathfrak{g}(A \cup\{x, x+1, x+3\}) \\
& +\sum \sum[\mathfrak{f}(A \cup\{x+1, x+3\})-\mathfrak{f}(A \cup\{x, x+1\})] \mathfrak{g}(A \cup\{x, x+1, x+3\}),
\end{aligned}
$$

where the first sum in each line is for $x \neq 0,-1,-3$ and the second one is for $A \in \mathcal{E}_{*, n-3}$ such that $A \cap\{x, x+$ $1, x+3\}=\emptyset$. Then $T_{2}$ vanishes too. Finally, for $T_{3}$, we get

$$
\begin{aligned}
T_{3}= & \sum \sum[\mathfrak{f}(A \cup\{x\})-\mathfrak{f}(A \cup\{x+1\})] \mathfrak{g}(A \cup\{x, x+1\}) \\
& +\sum \sum[\mathfrak{f}(A \cup\{x+1\})-\mathfrak{f}(A \cup\{x+3\})] \mathfrak{g}(A \cup\{x+1, x+3\}) \\
& +\sum \sum[\mathfrak{f}(A \cup\{x+3\})-\mathfrak{f}(A \cup\{x\})] \mathfrak{g}(A \cup\{x, x+3\}),
\end{aligned}
$$

where the first sum in each line is for $x \neq 0,-1,-3$ and the second one is for $A \in \mathcal{E}_{*, n-3}$ such that $A \cap\{x, x+$ $1, x+3\}=\emptyset$. Add and subtract $\mathfrak{f}(A \cup\{x+1\})$ in the first factor of the last line in (B.11) to get that

$$
\begin{aligned}
T_{3}= & \sum \sum[\mathfrak{f}(A \cup\{x+3\})-\mathfrak{f}(A \cup\{x+1\})][\mathfrak{g}(A \cup\{x, x+3\})-\mathfrak{g}(A \cup\{x+1, x+3\})] \\
& +[\mathfrak{f}(A \cup\{x+1\})-\mathfrak{f}(A \cup\{x\})][\mathfrak{g}(A \cup\{x, x+3\})-\mathfrak{g}(A \cup\{x, x+1\})] .
\end{aligned}
$$
(B.3).

By Schwarz inequality, the previous expression is bounded by $C\left\langle\mathfrak{f},-\mathfrak{L}_{s}^{\pi}, \mathfrak{f}\right\rangle\left\langle\mathfrak{g},-\mathfrak{L}_{s}^{\pi}, \mathfrak{g}\right\rangle$, in view of formula

We turn now to the expression $\sum_{i=1}^{3} M_{a_{i}}$ (see (B.8) for definition of $M_{b}$ ). It may be rewritten as

$$
\begin{aligned}
\sum_{i=1}^{3} \sum_{A \cap\left\{0, a_{i}\right\}=\emptyset}\left[\mathfrak{f}(A)-\mathfrak{f}\left(S_{a_{i}} A\right)\right] \mathfrak{g}\left(A \cup\left\{a_{i}\right\}\right)= & \sum_{A \cap\{0,1\}=\emptyset}\left[\mathfrak{f}(A)-\mathfrak{f}\left(S_{1} A\right)\right] \mathfrak{g}(A \cup\{1\}) \\
& +\sum_{A \cap\{1,3\}=\emptyset}\left[\mathfrak{f}\left(S_{1} A\right)-\mathfrak{f}\left(S_{3} A\right)\right] \mathfrak{g}\left(S_{1} A \cup\{2\}\right) \\
& -\sum_{A \cap\{0,3\}=\emptyset}\left[\mathfrak{f}(A)-\mathfrak{f}\left(S_{3} A\right)\right] \mathfrak{g}\left(S_{3} A \cup\{-3\}\right),
\end{aligned}
$$

where the last two terms in the previous expression are obtained after the change of variable $S_{2} A=B$ and $S_{-3} A=B$, respectively. We decompose each expression in two, to obtain sums carried over the same sets. In the following expression, the first three terms correspond to the terms obtained by imposing $A$ not to contain 3 , 0 and 1, respectively in each of the three last lines of (B.12). The sum over the sets $A$ that contain 3,0 and 1 , correspond to the last three lines. Therefore,

$$
\begin{aligned}
\sum_{i=1}^{3} M_{a_{i}}= & \sum_{A \cap\{0,1,3\}=\emptyset}\left[\mathfrak{f}(A)-\mathfrak{f}\left(S_{1} A\right)\right] \mathfrak{g}(A \cup\{1\})+\left[\mathfrak{f}\left(S_{1} A\right)-\mathfrak{f}\left(S_{3} A\right)\right] \mathfrak{g}\left(S_{1} A \cup\{2\}\right) \\
& -\left[\mathfrak{f}(A)-\mathfrak{f}\left(S_{3} A\right)\right] \mathfrak{g}\left(S_{3} A \cup\{-3\}\right)+\left[\mathfrak{f}(A \cup\{3\})-\mathfrak{f}\left(S_{1} A \cup\{2\}\right)\right] \mathfrak{g}(A \cup\{1,3\}) \\
& +\left[\mathfrak{f}\left(S_{1} A \cup\{-1\}\right)-\mathfrak{f}\left(S_{3} A \cup\{-3\}\right)\right] \mathfrak{g}\left(S_{1} A \cup\{2,-1\}\right) \\
& -\left[\mathfrak{f}(A \cup\{1\})-\mathfrak{f}\left(S_{3} A \cup\{-2\}\right)\right] \mathfrak{g}\left(S_{3} A \cup\{-3,-2\}\right) .
\end{aligned}
$$

After some operations recalling the definition of $S_{z}$ and the fact that $\mathfrak{f}$ and $\mathfrak{g}$ belong to $\mathcal{I}_{*}$ (as we did when working with $T_{1}$ ), we get that the sum of the last three terms vanishes. For the three remaining, add and subtract $\mathfrak{f}\left(S_{1} A\right)$ in the third line, to get 


$$
\begin{aligned}
& \sum_{A \cap\{0,1,3\}=\emptyset}\left[\mathfrak{f}(A)-\mathfrak{f}\left(S_{1} A\right)\right][\mathfrak{g}(A \cup\{1\})-\mathfrak{g}(A \cup\{3\})] \\
& \quad+\sum_{A \cap\{0,1,3\}=\emptyset}\left[\mathfrak{f}\left(S_{1} A\right)-\mathfrak{f}\left(S_{3} A\right)\right]\left[\mathfrak{g}\left(S_{1}(A \cup\{3\})\right)-\mathfrak{g}\left(S_{3}(A \cup\{3\})\right)\right] .
\end{aligned}
$$

By Schwarz inequality, this expression is bounded by $C\|\mathfrak{f}\|_{1}\|\mathfrak{g}\|_{1}$, in view of expression (B.3) for $\|\cdot\|_{1}$. This concludes the proof of the lemma.

Lemma B.7. Given a loop probability $\pi$ there exists a finite constant $C_{0}$, depending only on the probability $\pi$, such that

$$
\left\langle\mathfrak{g}, \mathfrak{L}_{-}^{\pi} \mathfrak{f}\right\rangle^{2} \leqslant C_{0}\left\langle\mathfrak{f}, \mathfrak{L}_{s}^{\pi} \mathfrak{f}\right\rangle\left\langle\mathfrak{g}, \mathfrak{L}_{s}^{\pi} \mathfrak{g}\right\rangle,
$$

for each $\mathfrak{f} \in \mathcal{I}_{n+1}, \mathfrak{g} \in \mathcal{I}_{n}$.

Proof. This result follows from Lemmas B.1 and B.6.

Lemma B.8. Given a loop probability $\pi$ there exists a finite constant $C_{0}$, depending only on the probability $\pi$, such that

$$
\left\langle\mathfrak{g}, \mathfrak{L}_{d}^{\pi} \mathfrak{f}\right\rangle^{2} \leqslant C_{0}\left\langle\mathfrak{f}, \mathfrak{L}_{s}^{\pi} \mathfrak{f}\right\rangle\left\langle\mathfrak{g}, \mathfrak{L}_{s}^{\pi} \mathfrak{g}\right\rangle
$$

for each $\mathfrak{f}, \mathfrak{g} \in \mathcal{I}_{n}$.

Proof. We follow the strategy used in the previous case. Observe that

$$
\left\langle\mathfrak{g}, \mathfrak{L}_{d}^{\pi} \mathfrak{f}\right\rangle=1 /(2 m) \sum_{i=1}^{m} N_{a_{i}}+1 /(2 m) \sum_{i=1}^{m} M_{a_{i}},
$$

where

$$
\begin{aligned}
N_{b} & =\sum_{x \neq 0,-b} \sum_{\substack{A \in \mathcal{E}_{*, n-1} \\
A \cap\{x, x+b\}=\emptyset}}[\mathfrak{f}(A \cup\{x+b\})-\mathfrak{f}(A \cup\{x\})][\mathfrak{g}(A \cup\{x\})+\mathfrak{g}(A \cup\{x+b\})], \\
M_{b} & =\sum_{\substack{A \in \mathcal{E}_{*, n} \\
A \cap\{b\}=\emptyset}}\left[\mathfrak{f}\left(S_{b} A\right)-\mathfrak{f}(A)\right]\left[\mathfrak{g}(A)+\mathfrak{g}\left(S_{b} A\right)\right] .
\end{aligned}
$$

This decomposition allows us to repeat the same kind of computation performed when proving Lemma B.6.

\section{References}

[1] C. Bernardin, Regularity of the diffusion coefficient for lattice gas reversible under Bernoulli measures, Stochastic Process. Appl. 101 (1) (2002) 43-68.

[2] C. Kipnis, C. Landim, Scaling Limits of Interacting Particle Systems, Grundlehren der Mathematischen Wissenschaften (Fundamental Principles of Mathematical Sciences), vol. 320, Springer-Verlag, Berlin, 1999.

[3] K. Komoriya, Hydrodynamic limit for asymmetric mean zero exclusion processes with speed change, Ann. Inst. H. Poincaré Probab. Statist. 34 (6) (1998) 767-797.

[4] C. Landim, Conservation of local equilibrium for attractive particle systems on $\mathbb{Z}^{d}$, Ann. Probab. 21, 1782-1808.

[5] C. Landim, S. Olla, H.T. Yau, Some properties of the diffusion coefficient for asymmetric simple exclusion processes, Ann. Probab. 24 (1996) 1779-1807.

[6] C. Landim, S. Olla, S.R.S. Varadhan, Asymptotic behavior of a tagged particle in simple exclusion processes, Bol. Soc. Bras. Mat. 31 (2000) 241-275. 
[7] C. Landim, S. Olla, S.R.S. Varadhan, On viscosity and fluctuation-dissipation in exclusion processes, preprint.

[8] C. Landim, S. Olla, S.R.S. Varadhan, Symmetric simple exclusion process: regularity of the self-diffusion coefficient, Comm. Math. Phys. 224 (2001) 307-321.

[9] C. Landim, H.T. Yau, Fluctuation-dissipation equation of asymmetric simple exclusion process, Probab. Theory Related Fields 108 (1997) $321-356$

[10] J. Quastel, Diffusion of color in the simple exclusion process, Comm. Pure Appl. Math. XLV, 623-679.

[11] S. Sethuraman, S.R.S. Varadhan, H.T. Yau, Diffusive limit of a tagged particle in asymmetric exclusion process, Comm. Pure Appl. Math. 53 (2000) 972-1006.

[12] S.R.S. Varadhan, Non-linear diffusion limit for a system with nearest neighbor interactions II, in: K.D. Elworthy, N. Ikeda (Eds.), Asymptotic Problems in Probability Theory, Stochastic Models and Diffusion on Fractals, in: Pitman Research Notes in Mathematics, vol. 283, Wiley, New York, 1994, pp. 75-128.

[13] S.R.S. Varadhan, Selfdiffusion of a tagged particle in equilibrium for asymmetric mean zero random walk with simple exclusion, Ann. Inst. Henri Poincaré 31 (1) (1995) 273-285.

[14] L. Xu, Diffusion limit for the lattice gas with short range interactions, PhD Thesis, New York University, 1993.

[15] H.T. Yau, Relative entropy and hydrodynamics of Ginzburg-Landau models, Lett. Math. Phys. 22, 63-80. 